DESY 03-120

\title{
Universality and scaling behavior of RG gauge actions
}

\author{
Silvia Necco \\ DESY, Platanenallee 6, D-15738 Zeuthen, Germany \\ necco@ifh.de
}

\begin{abstract}
We study universality and scaling properties of RG gauge actions (Iwasaki and DBW2). In the first part we consider the critical temperature $T_{c}$ and compute the reference energy scale $r_{0}$ for critical couplings $\beta_{c}$ corresponding to $N_{t}=$ $3,4,6,8$. The universality of $T_{c} r_{0}$ between Iwasaki and Wilson action is confirmed and the scaling behavior of the Iwasaki action is found to be better than the one for the Wilson action. The results for the DBW2 action show larger lattice artefacts. A continuum value $T_{c} r_{0}=0.7498(50)$ is extracted. We compute also the glueball masses for the states $0^{++}$and $2^{++}$, investigate the scaling of $m_{0^{+}+} r_{0}$ and $m_{2^{+}+} r_{0}$ and point out practical problems which are due to the violation of positivity present in the RG actions.
\end{abstract}




\section{Introduction}

In view of the next unquenched lattice simulations, big efforts were devoted in the last years to improve both fermionic and gauge actions.

Although the lattice artefacts for the standard gauge action start at $\mathrm{O}\left(a^{2}\right)$ and hence one expects that these are somehow less relevant than the ones induced by the fermionic part, it turns out that the gauge term plays an important rôle, and the question which is the most convenient gauge action to adopt has been often addressed.

The purpose of adopting alternative actions is not only to improve the scaling behavior; also features related to chiral symmetry were investigated. In particular, RG actions (Iwasaki, DBW2) have been suggested as good candidates to be used in the next simulations on Ginsparg-Wilson/domain wall fermions; interesting characteristics were observed, such as the suppression of small instantons and dislocations and a possible remedy of the problem of residual chiral symmetry breaking for domain wall fermions [1,2]. Other authors [3], however, pointed out possible problems connected to these actions and proposed an alternative method to reduce residual-mass effects that works very well also with the Wilson plaquette action.

This increasing interest and discussion in improved gauge actions motivates more investigations into their properties, starting from the basic ones, like universality and scaling behavior.

There are in principle several quantities that one can use to quantify the lattice artefacts and to test universality by comparing the results with the plaquette action known in the literature; in particular in this work we will consider the critical deconfining temperature $T_{c}$ and the glueball masses for the states $0^{++}$and $2^{++}$.

In a previous work [4], the scaling of the ratio $T_{c} / \sqrt{\sigma}$ has been investigated and lead for the Iwasaki action to a continuum limit which is in disagreement with the one obtained with the Wilson action. More likely than a possible violation of universality, this fact is related to the definition of the string tension $\sigma$. We will perform the same analysis but using the scale $r_{0} \approx 0.5 \mathrm{fm}[5]$ instead of $\sigma$. It is well known that the extraction of the string tension from the static potential at finite quark separations is problematic and leads to systematic errors, which are difficult to control. It is preferable to define the scale by using intermediate distance properties.

After computing $r_{0} / a$ at several lattice spacing for Iwasaki and DBW2 actions we will perform a continuum extrapolation of the quantity $T_{c} r_{0}$, discuss universality and compare the scaling violations for the different actions. 
We will point out possible problems that can occur by adding irrelevant operators to the plaquette action, as the violation of physical positivity.

The second part of the work is dedicated to the evaluation of the glueball masses. In particular the $0^{++}$mass is a promising observable to discuss the lattice artefacts, since for the Wilson action large scaling violations have been observed.

Despite the large errors and the difficulties in the calculations, we will discuss our results, draw indicative conclusions and remark on open questions.

\section{Improved gauge actions}

In this work we will consider SU(3) Yang-Mills lattice actions formulated on the basis of renormalization group ( $\mathrm{RG}$ ) considerations. The most popular examples are the Iwasaki [6] and DBW2 [7,8] actions, which are restricted to a twoparameter space and include planar rectangular $(1 \times 2)$ loops in addition to the usual plaquette term,

$$
S=\beta \sum_{x}\left(c_{0} \sum_{\mu<\nu}\left\{1-\frac{1}{3} \operatorname{Re} W_{\mu \nu}^{1 \times 1}(x)\right\}+c_{1} \sum_{\mu, \nu}\left\{1-\frac{1}{3} \operatorname{Re} W_{\mu \nu}^{1 \times 2}(x)\right\}\right),
$$

with the normalization condition $c_{0}=1-8 c_{1}$.

The coefficient $c_{1}$ in eq. (2.1) takes different values for various choices of alternative actions

$$
c_{1}= \begin{cases}-1 / 12 & \text { Symanzik, tree level impr. } \\ -0.331 & \text { Iwasaki, RG } \\ -1.4088 & \text { DBW2, RG }\end{cases}
$$

where we included also the $\mathrm{O}\left(a^{2}\right)$ tree-level improved Symanzik action [9], [10]. First of all one can notice that the strength of the rectangular loops for RG improved actions is significantly larger than what is needed in order to cancel the $\mathrm{O}\left(a^{2}\right)$ effects at tree level. We will later discuss this further.

We will also consider the gauge FP action [11] for comparison, in particular the study on the lattice artefacts performed in [12].

\section{The critical temperature $T_{c}$}

It is well known $[13,14]$ that pure Yang-Mills theory undergoes a first order phase transition at some finite temperature $T_{c}$. On the lattice, the critical temperature is determined by evaluating the critical coupling $\beta_{c}$

$$
\frac{1}{T_{c}}=N_{t} a\left(\beta_{c}\right)
$$




\begin{tabular}{|c|c|c|c|}
\hline$N_{t}$ & Wilson [16] & Iwasaki [4] & $\overline{\text { DBW2 [17] }}$ \\
\hline 3 & & $2.1551(12)$ & $0.75696(98)$ \\
\hline 4 & $5.6925(2)$ & $2.2879(11)$ & $0.82430(95)$ \\
\hline 6 & $5.8941(5)$ & $2.5206(24)$ & $0.9636(25)$ \\
\hline 8 & $6.0624(12)$ & $2.7124(34)$ & \\
\hline 12 & $6.3380(23)$ & & \\
\hline
\end{tabular}

Table 1: The critical coupling for Wilson, Iwasaki and DBW2 actions.

where $N_{t}$ is the number of lattice points in the time-like direction with periodic boundary conditions. The extension in the space-like directions is supposed to be infinite.

There are several methods for determining $\beta_{c}$; for example, one can locate the peak in the Polyakov loop susceptibility.

In addition to its intrinsic importance as a fundamental non-perturbative prediction, $T_{c}$ provides also a useful quantity to study the lattice artefacts for different gauge actions and to test universality.

For known values of $\beta_{c}$ at given $N_{t}$ for different actions one can refer to $[15,16,4,17$, 12]; for Wilson, Iwasaki and DBW2 actions, these values are collected in Table 1.

\begin{tabular}{ccc}
\hline action & $N_{t}$ & $T_{c} / \sqrt{\sigma}$ \\
\hline Wilson [16] & $\infty$ & $0.630(5)$ \\
Iwasaki [4] & $\infty$ & $0.651(12)$ \\
DBW2 [17] & $\infty$ & $0.627(12)$ \\
Sym. tree level [16] & $\infty$ & $0.634(8)$ \\
1 loop tadpole impr. [18] & $\infty$ & $0.659(8)$ \\
FP [12] & 4 & $0.624(7)$ \\
\hline
\end{tabular}

Table 2: Results for the deconfining temperature in units of the string tension from different actions. The continuum extrapolations are taken from [19].

The available results in the literature are mostly expressed in terms of the string tension $\sigma$; the results for the quantity $T_{c} / \sqrt{\sigma}$ are reported in Table 2 , where the continuum extrapolations are from [19]. Figure 1 collects the results for Wilson, Iwasaki, DBW2, Symanzik tree level and FP action, and was taken from [12], where the latest evaluation of $T_{c} / \sqrt{\sigma}$ (FP action) has been performed.

The first observation from Fig. 1 is that for this specific quantity the dis- 


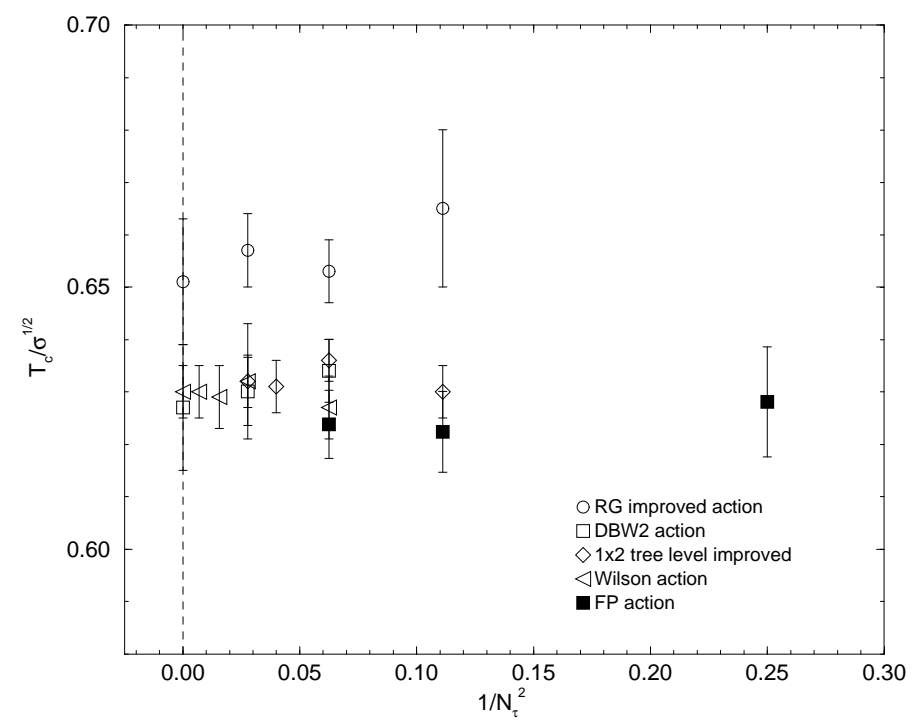

Figure 1: $T_{c} / \sqrt{\sigma}$ as function of $1 / N_{t}^{2}$ for different actions, from [12]. By "RG improved" is meant here the Iwasaki action.

cretization effects appear not to be very significant and hence is not possible to arrive at precise conclusions about the lattice artefacts for different actions.

Furthermore, one notices that for the Iwasaki and the Wilson actions a difference of order $2 \sigma$ in the continuum results is observed. The most drastic explanation for this discrepancy could be a violation of universality, but this scenario seems unrealistic; a more natural explanation is that the string tension is difficult to determine and systematic errors due to this were not included in all calculations. It is preferable to use $r_{0}[5]$ to reliably set the scale.

Let us recall that the string tension is related to the properties of the force between static quarks for distances $r \rightarrow \infty$, and hence its evaluation at finite $r$ can in principle contain large systematic errors.

On the contrary, $r_{0} \approx 0.5 \mathrm{fm}$ is extracted from the force at intermediate distances and can be evaluated very precisely. For the Wilson action this quantity has been evaluated in the coupling range $5.7 \leq \beta \leq 6.92[20,21]$.

\section{Evaluation of $r_{0} / a$ for RG actions}

For the Wilson action, the values of $r_{0} / a$ corresponding to the critical couplings at different $N_{t}$ can be easily obtained by the parametrization formula in [20].

For the Iwasaki and DBW2 actions there was up to now no precise evaluation of 
$r_{0} / a$ and we performed new numerical simulations with this purpose.

In our computation we followed essentially the procedure adopted in $[5,20]$ for the plaquette action. We applied the smearing procedure [22] to the spatial links and the multi-hit method [23] to the time-like links for the variance reduction.

For each spatial separation $r$ we constructed Wilson loop correlation matrices

$$
C_{l m}(t)=\left\langle\operatorname{Tr}\left\{V_{l}(0, r \hat{1}) \bar{V}(r \hat{1}, r \hat{1}+t \hat{0}) V_{m}^{\dagger}(t \hat{0}, r \hat{1}+t \hat{0}) \bar{V}^{\dagger}(0, t \hat{0})\right\}\right\rangle
$$

with $l, m=1, \ldots, M ; V_{l}(x, y)$ indicates the product of link variables connecting $x$ and $y$ at smearing level $l$ in a spatial direction, while $\bar{V}(x, y)$ is the product of time-like links between $x$ and $y$ after the application of the multihit procedure. In particular we have chosen $M=4$, and the number of smearing iterations for each level has been determined with the same criterion as for the Wilson action [20].

We adopted a hybrid algorithm with $N_{\text {or }}$ over-relaxation steps per heat-bath step [24,25] and increased $N_{\text {or }}$ with $\beta$ according to

$$
N_{\text {or }} \approx 1.5\left(r_{0} / a\right)
$$

The simulation parameters are reported in Table 3. For the DBW2 action, besides the three values of $\beta_{c}$ known in the literature (Table 1 ), we decided to evaluate $r_{0} / a$ also for a larger $\beta=1.04$, which should roughly correspond to $\beta=6$ for the Wilson action and has been used in quenched simulations [2].

We started our analysis by observing the time-dependence of the effective potential evaluated from the diagonal elements of the correlation matrices.

Figure 2 shows the effective potential

$$
a V(r)=-\ln \left(\frac{C_{22}(t)}{C_{22}(t-a)}\right)
$$

evaluated for $l=m=2$, which corresponds to what was estimated to be the optimal smearing for the Wilson action in [26].

It has been pointed out in [27] (see app. A) that actions containing terms in addition to the plaquette violate the physical positivity, and hence one expects also negative contributions to the spectral decomposition of correlation functions. These negative contributions for the RG actions are evident in Fig. 2 and, as expected from our discussion in app. A, they are invisible starting from a certain $t$ which depends on the coefficient $c_{1}$ of eq. (2.2).

The most important consequence of this phenomenon regards the applicability of the variational method $[28,29]$, which is mathematically founded on the positivity 


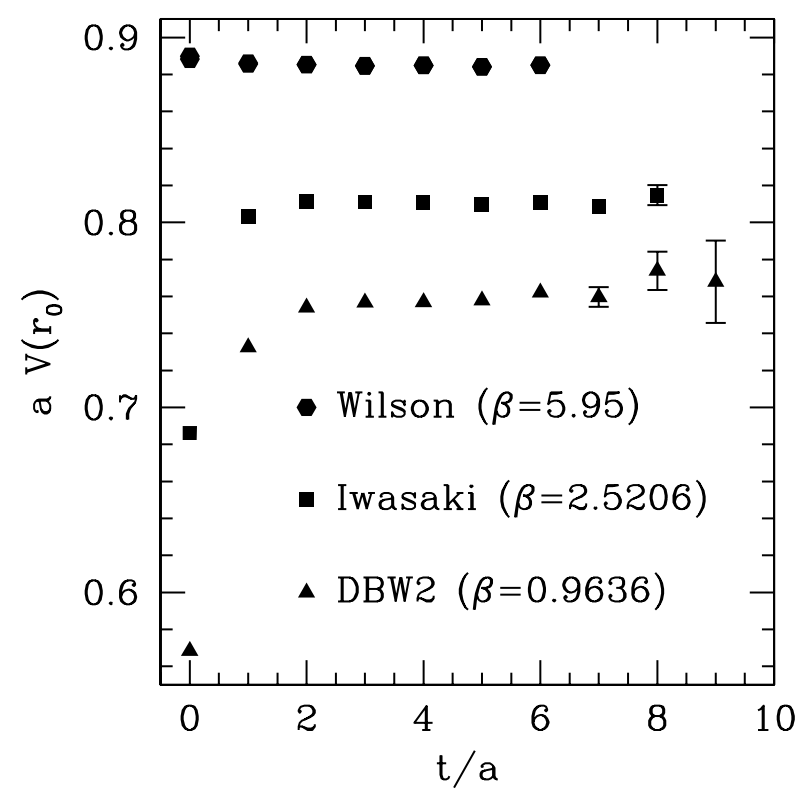

Figure 2: The effective potential as function of $t$ for $r \approx r_{0}\left(r_{0} / a \sim 4-5\right)$ for the Wilson action and for the RG-improved Iwasaki and DBW2 action.

of the correlation matrix $C_{l m}(t)$ at a certain small $t=t_{0}$; this condition is verified only for $t_{0} \gg t_{\text {min }}$, but on the other hand $t_{0}$ can not be arbitrarily large because the statistical errors increase exponentially with $t$ and make the inversion of $C_{l m}$ impracticable.

One observes however a quite satisfactory plateau in the effective potential, starting at sufficiently large $t$. In that sense the violation of physical positivity does not represent a real trouble for these correlation functions, but can become quite problematic for example for the extraction of the glueball masses (see sec. 7).

We decided to extract the potential from eq. (4.3) at $t / a=(3-4)$ without applying the variational method. The systematic error was estimated by taking the difference between this value and what one would obtain extracting the potential at $(t+a)$. We linearly added systematic and statistical errors; at small $r$ the total uncertainty is dominated by the systematic one, while at large distances the situation is in general reversed.

\subsection{The force at tree level}

A tree-level study of the force for the different actions can furnish important hints on how the continuum limit is approached, in particular in the region of small couplings. 


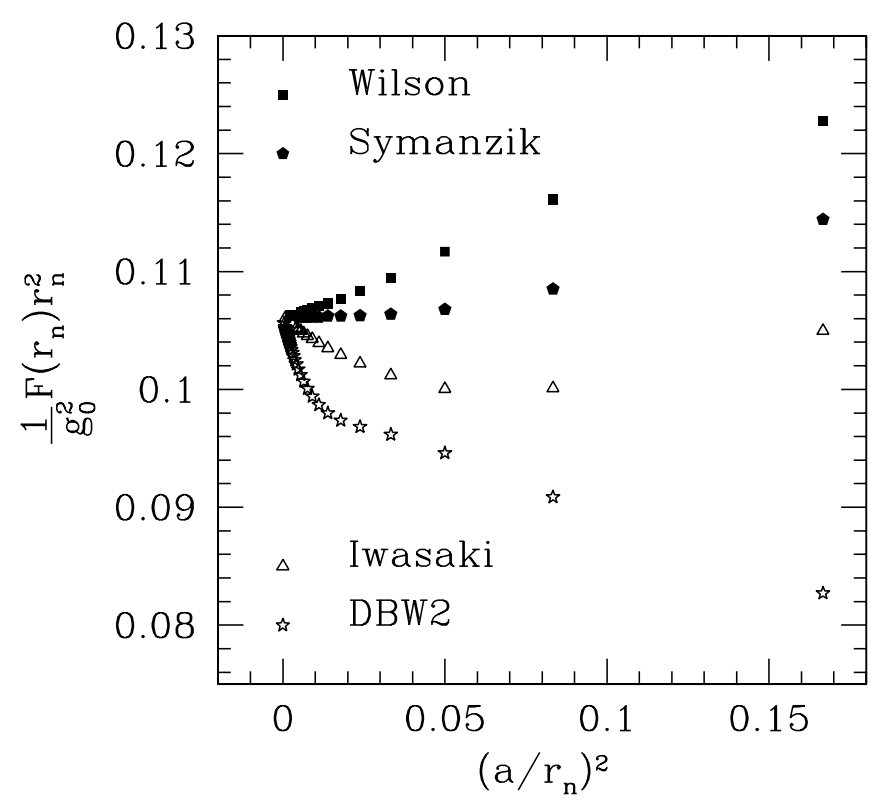

Figure 3: The force at tree level for several actions.

Explicitly, for the action eq. (2.1) the tree-level force is given by [9]

$$
\begin{gathered}
F\left(r^{\prime}\right)=\frac{V(r)-V(r-a)}{a}= \\
=-\frac{4}{3} \frac{g_{0}^{2}}{a} \int_{-\pi}^{\pi} \frac{d^{3} k}{(2 \pi)^{3}} \frac{\cos \left(r k_{1} / a\right)-\cos \left((r-a) k_{1} / a\right)}{4\left(\sum_{j=1}^{3} \sin ^{2}\left(k_{j} / 2\right)-4 c_{1} \sum_{j=1}^{3} \sin ^{4}\left(k_{j} / 2\right)\right)}+\mathrm{O}\left(g_{0}^{4} a^{2}\right)= \\
=F_{\text {tree }}\left(r^{\prime}\right)+\mathrm{O}\left(g_{0}^{4} a^{2}\right),
\end{gathered}
$$

where $g_{0}^{2}=6 / \beta$.

In Fig. 3 the quantity $\frac{r_{n}^{2}}{g_{0}^{2}} F_{\text {tree }}\left(r_{n}\right)$, with $r_{n}=r-a / 2$ is plotted as function of $\left(a / r_{n}\right)^{2}$ for different actions. One can notice that the RG actions, in particular the DBW2, show at tree level large lattice artefacts at small $\left(a / r^{\prime}\right)$. In that sense, at tree level the RG actions are "over-corrected" and introduce lattice artefacts of the same order or even larger than what is expected with the usual plaquette action. This fact indicates that the continuum extrapolation should be considered with great care, because unless $\left(a / r^{\prime}\right)$ is very small, one can not be sure to be in the region where the leading discretization errors are quadratic in $a$.

Beside the naive definition of the force

$$
F\left(r^{\prime}\right)=[V(r)-V(r-a)] / a,
$$

with $r^{\prime}=r_{n}$, we followed the procedure reported in [5] by introducing a tree-level improved definition with $r^{\prime}=r_{\text {I }}$ such that

$$
F_{\text {tree }}\left(r_{\mathrm{I}}\right)=\frac{4}{3} \frac{g_{0}^{2}}{4 \pi r_{\mathrm{I}}^{2}},
$$


with no lattice artefacts at tree level. For the Wilson action this choice turned out to improve substantially the scaling behavior of the force $[5,20]$.

From eq. (4.4) one obtains

$$
\left(4 \pi r_{\mathrm{I}}^{2}\right)^{-1}=-[G(r, 0,0)-G(r-a, 0,0)] / a,
$$

where

$$
G(\vec{r})=\frac{1}{a} \int_{-\pi}^{\pi} \frac{d^{3} k}{(2 \pi)^{3}} \frac{\prod_{j=1}^{3} \cos \left(r_{j} k_{j} / a\right)}{4\left(\sum_{j=1}^{3} \sin ^{2}\left(k_{j} / 2\right)-4 c_{1} \sum_{j=1}^{3} \sin ^{4}\left(k_{j} / 2\right)\right)}
$$

is the scalar free lattice propagator associated to the action eq. (2.1). We computed $r_{\text {I }}$ by solving the integral eq. (4.8) numerically.

Iwasaki action:

\begin{tabular}{ccccc}
\hline$L / a$ & $\beta$ & $n_{l}$ & $N_{\text {or }}$ & $N_{\text {meas }}$ \\
\hline 8 & 2.1551 & $0,2,4,6$ & 3 & 20000 \\
12 & 2.2879 & $0,4,9,13$ & 4 & 4000 \\
24 & 2.5206 & $0,12,25,37$ & 8 & 645 \\
32 & 2.7124 & $0,18,36,54$ & 9 & 370 \\
\hline
\end{tabular}

DBW2 action :

\begin{tabular}{ccccc}
\hline$L / a$ & $\beta$ & $n_{l}$ & $N_{\text {or }}$ & $N_{\text {meas }}$ \\
\hline 10 & 0.75696 & $0,2,4,6$ & 3 & 12000 \\
12 & 0.8243 & $0,4,9,13$ & 4 & 6000 \\
16 & 0.9636 & $0,10,20,30$ & 8 & 800 \\
24 & 1.04 & $0,18,36,54$ & 9 & 220 \\
\hline
\end{tabular}

Table 3: Simulation parameters for the Iwasaki and DBW2 actions for the evaluation of $r_{0} / a$. $L$ is the lattice extension (the spatial and temporal extensions are equal), $n_{l}$ represents the number of smearing iterations for each level.

\subsection{Results}

Our numerical results for the potential and the force at finite lattice spacing are collected in the tables 11 and 12 in App. B.

Once the force has been evaluated, we extracted the value of $r_{0} / a$ by using a local interpolation formula; the results are reported in Table 4. We adopted both the naive and the tree-level improved definition of the force.

The first error contains the statistical uncertainty summed to the systematic one due to the interpolation of the force. The second error is the systematic 
Iwasaki action :

\begin{tabular}{cccc}
\hline$\beta$ & $r_{0} / a\left(r_{n}\right)$ & $r_{0} / a\left(r_{\mathrm{I}}\right)$ & $r_{0} / a($ eq. $(4.9))$ \\
\hline 2.1551 & $2.311(5)(9)$ & $2.320(6)(9)$ & 2.333 \\
2.2879 & $3.026(4)(3)$ & $3.026(5)(1)$ & 3.021 \\
2.5206 & $4.535(6)(4)$ & $4.511(8)(1)$ & 4.514 \\
2.7124 & $6.020(15)(25)$ & $5.999(15)(19)$ & 5.983 \\
\hline
\end{tabular}

DBW2 action :

\begin{tabular}{ccc}
\hline$\beta$ & $r_{0} / a\left(r_{n}\right)$ & $r_{0} / a\left(r_{\mathrm{I}}\right)$ \\
\hline 0.75696 & $2.430(5)(20)$ & $2.225(4)(11)$ \\
0.8243 & $3.129(23)(1)$ & $3.036(17)(4)$ \\
0.9636 & $4.606(13)(17)$ & $4.556(17)(20)$ \\
1.04 & $5.500(29)(7)$ & $5.452(26)(8)$ \\
\hline
\end{tabular}

Table 4: Results for $r_{0} / a$ evaluated at different $\beta=\beta_{c}$ for Iwasaki and DBW2 actions, using the naive definition of the force or the tree-level improved eq. (4.7). For the Iwasaki action, the fourth column is obtained from the fits eq. (4.9).

uncertainty coming from different choices of $t$ in the effective potential eq. (4.3). One can notice that for the DBW2 action the choice of $r_{n}$ or $r_{\mathrm{I}}$ in the definition of the force leads to results which can be quite different from each other, above all at small $r_{0} / a$; we expected this feature by investigating the force at tree level. This ambiguity will make the discussion of the lattice artefacts difficult, because the possible conclusions will depend on which definition of the force one has used and not on intrinsic properties of the action.

For the Iwasaki action the results obtained through the two definitions are not significantly different.

\subsection{Parametrization of $r_{0} / a$}

Following the strategy of [20], one can attempt a phenomenological parametrization of $r_{0} / a$ in the range of couplings under consideration.

For the Iwasaki action the four values of $r_{0}$ (obtained by adopting $r_{\mathrm{I}}$ ) were fitted in the form

$$
\ln \left(a / r_{0}\right)=c_{1}+c_{2}(\beta-3)+c_{3}(\beta-3)^{2},
$$

yielding the numerical results

$$
c_{1}=-2.1281, \quad c_{2}=-1.0056, \quad c_{3}=0.6041 .
$$




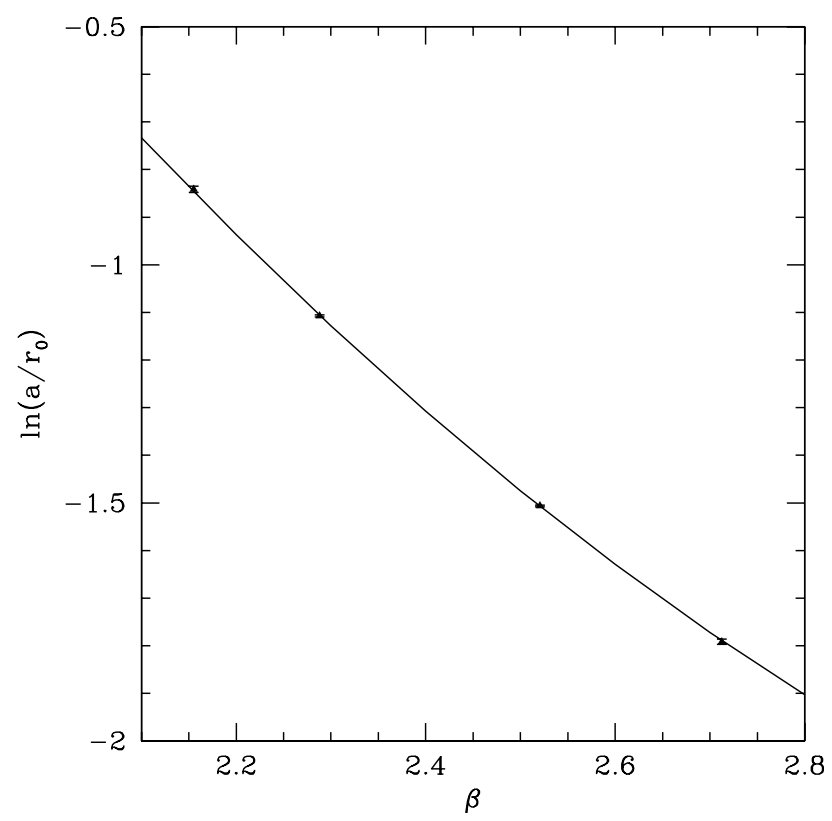

Figure 4: Parametrization of $r_{0} / a$ for the Iwasaki action, eq. (4.9), using the tree-level improved definition of the force.

in the range $2.1551 \leq \beta \leq 2.7124$.

The results and the fit formula are shown in Fig. 4; the accuracy is about $0.6 \%$ at $\beta=2.1551$ and $0.8 \%$ at $\beta=2.7124$.

For the DBW2 action we had to use a four-parameter representation

$$
\ln \left(a / r_{0}\right)=d_{1}+d_{2}(\beta-1)+d_{3}(\beta-1)^{2}+d_{4}(\beta-1)^{3}
$$

with

$$
d_{1}=-1.6007, \quad d_{2}=-2.3179, \quad d_{3}=-0.8020, \quad d_{4}=-19.8509,
$$

for the range $0.75696 \leq \beta \leq 1.04$, where the results always refer to the tree-level improved definition of the force. The parametrization is plotted in Fig. 5.

Note that in [2] the value $r_{0} / a=5.24(3)$ for the DBW2 action at $\beta=1.04$ is quoted. Our value differs about $3 \%$ from that one.

\section{Scaling of $T_{c} r_{0}$}

Once $r_{0} / a$ at the given couplings is known, one can finally consider the renormalized quantity

$$
r_{0} T_{c}=\frac{1}{N_{t}} \frac{r_{0}}{a}\left(\beta_{c}\right)
$$




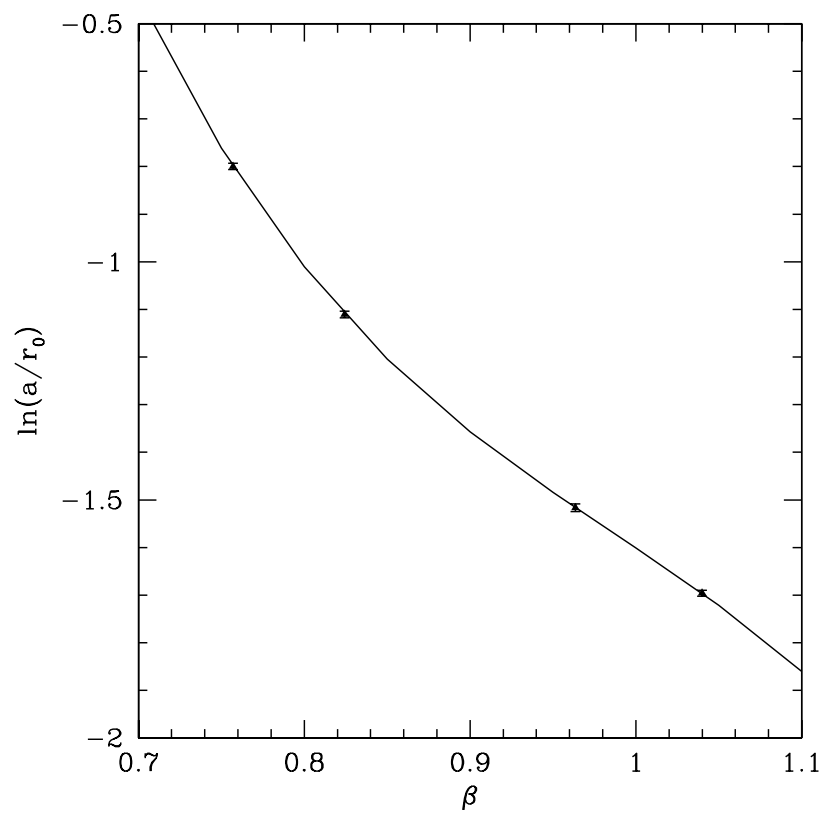

Figure 5: Parametrization of $r_{0} / a$ for the DBW2 action, eq. (4.11), using the tree-level improved definition of the force.

The results for Iwasaki and DBW2 actions are given in Table 5, together with the values obtained using the Wilson action. Also in this case, we show both the results obtained with the naive and with the tree-level improved definition of the force. The error in $T_{c} r_{0}$ is the quadratic sum of the error for $r_{0} / a$ and the uncertainty in $\beta_{c}$, which can be translated into an uncertainty in $r_{0}$ by using the parametrization formulas eq. (4.9), eq. (4.11). In our evaluations the error for $\beta_{c}$ and the uncertainty in $r_{0}$ are roughly of the same order.

We expect that the leading lattice artefacts are of order $a^{2}$, such that the continuum limit is approached in the following way

$$
T_{c} r_{0}=\left.T_{c} r_{0}\right|_{a=0}+s \cdot\left(a T_{c}\right)^{2}+\mathrm{O}\left(a T_{c}\right)^{4} .
$$

The results for $T_{c} r_{0}$, together with the continuum extrapolation, are shown in Fig. 6.

For the Iwasaki action there is no appreciable difference between the results obtained with $r_{n}$ and $r_{I}$ and in both cases the data show better scaling properties in comparison to the Wilson action. Furthermore, the value obtained at $N_{t}=8$ is in full agreement with the continuum result evaluated through the Wilson action and hence the universality is confirmed; this supports the conclusion that the disagreement observed in $T_{c} / \sqrt{\sigma}$ is indeed due to the difficulty in evaluating the string tension, particularly at small lattice spacings, and it is necessary to set the 


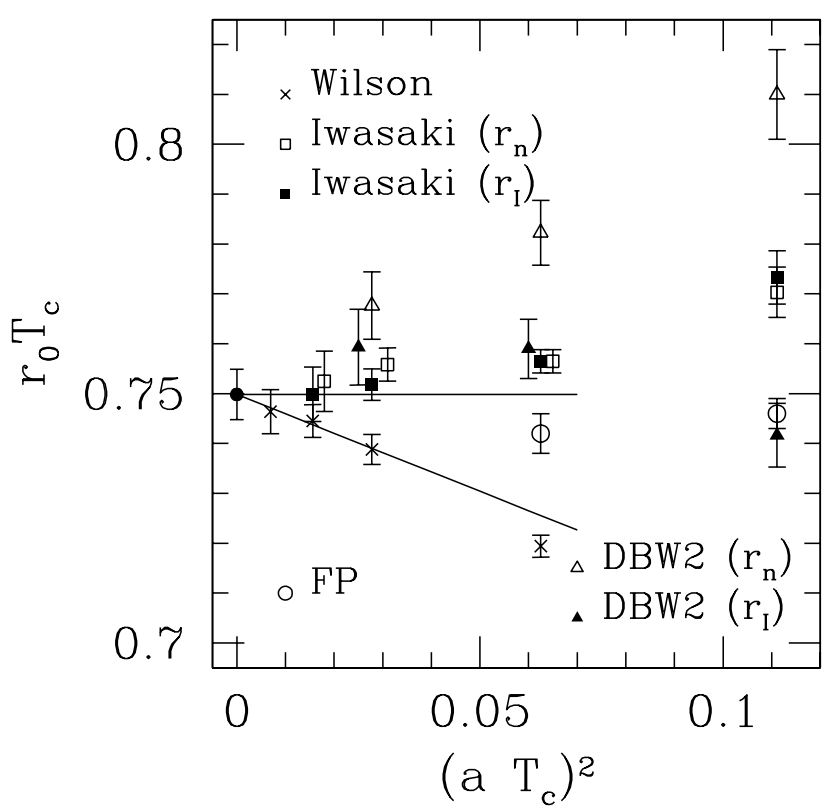

Figure 6: $T_{c} r_{0}$ for different actions. The $x$ coordinates were slightly shifted for clarity.

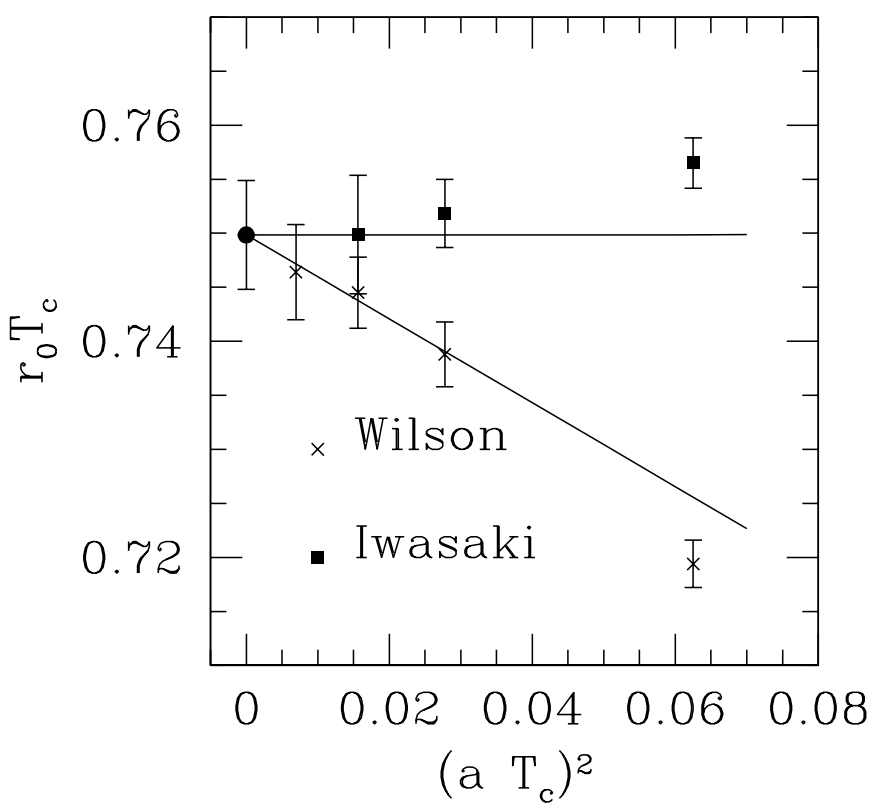

Figure 7: Continuum extrapolation of $T_{c} r_{0}$ for the Iwasaki and Wilson action, using the constrained fit eq. (5.2). 


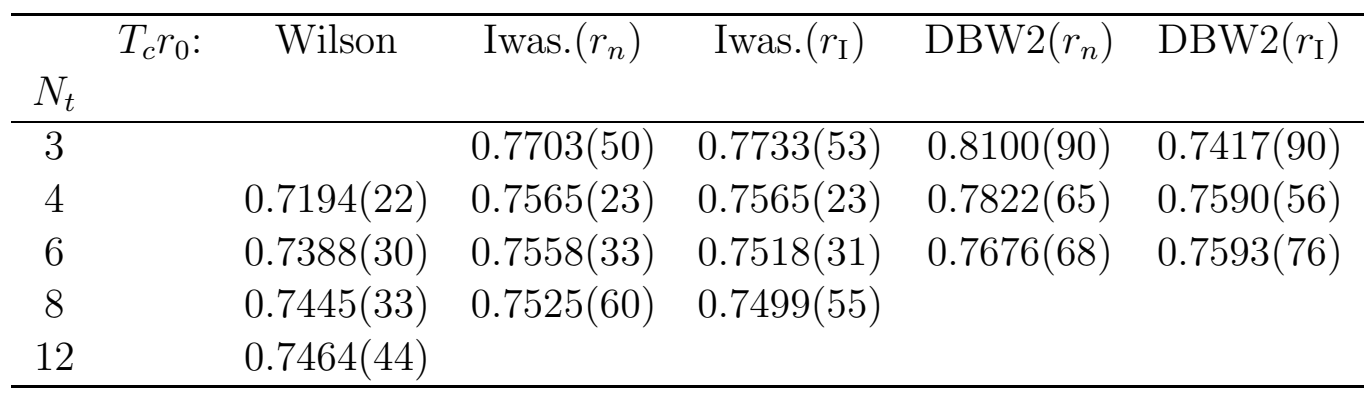

Table 5: Results for $T_{c} r_{0}$.

scale through a more reliable quantity.

Also for the DBW2 action the scaling properties are improved, although only by adopting $r_{I}$ instead of $r_{n}$, so that it is more difficult to make a statement about the lattice artefacts in this case.

A constrained fit of the form eq. (5.2) including the points with $N_{t} \geq 6$ for Iwasaki and Wilson actions yields the continuum result (Fig. 7)

$$
T_{c} r_{0}=0.7498(50) \text {. }
$$

At $N_{t}=6$ the Wilson action shows scaling violations for $r_{0} T_{c}$ of about $1.5 \%$, while they are $0.3 \%$ for the Iwasaki action.

For $N_{t}=4$ the discretization errors for the Wilson action increase to $4 \%$, while for the Iwasaki action they remain very small $(0.6 \%)$.

In Fig. 6 we included also the results obtained with the FP action [12], which also show a good scaling within $1 \%$ even on coarse lattices corresponding to $N_{t}=3,2$. One has however to mention that for those lattices the determination of $r_{0} / a$ contains large systematic uncertainties, as pointed out by the authors.

\section{Scaling of $\alpha_{\mathrm{q} \overline{\mathrm{q}}}(\mu)$}

Another interesting observable that can be used to test scaling violations is the dimensionless coupling $\alpha_{\mathrm{q} \overline{\mathrm{q}}}(\mu)$ obtained from the force. For this purpose we compared our present determination of the force at finite lattice spacing with the results of [21], where the continuum extrapolation has been performed in the region $0.05 \mathrm{fm} \leq r \leq 0.8 \mathrm{fm}$.

We point out that we only determined the on-axis potential and hence we can not investigate violations of rotational invariance which would require the evaluation of off-axis quantities. 


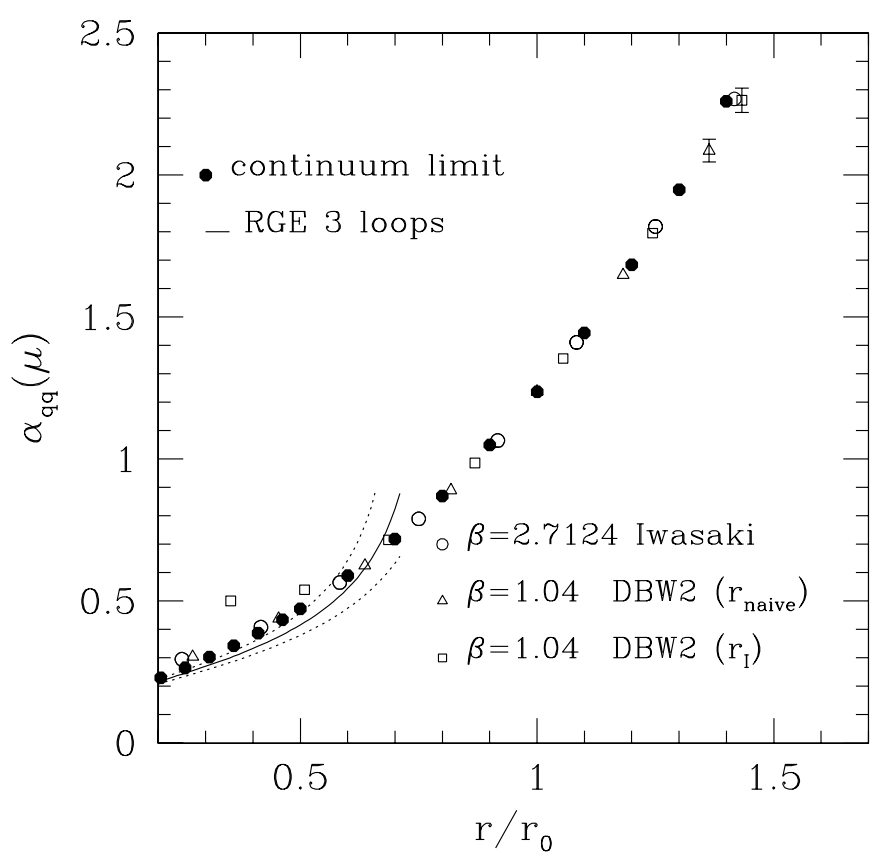

Figure 8: $\alpha_{\mathrm{qq}}$ at finite lattice spacing for Iwasaki and DBW2 action compared with the continuum result. The solid line represents the 3-loop RG perturbative prediction of the running coupling; the dashed lines correspond to its uncertainty [30].

The coupling $\alpha_{\mathrm{q} \overline{\mathrm{q}}}$ is defined in terms of the force by the simple relation

$$
\alpha_{\mathrm{q} \overline{\mathrm{q}}}(\mu)=\frac{3}{4} F(r) r^{2}, \quad \mu=\frac{1}{r} .
$$

Figure 8 shows $\alpha_{\mathrm{q} \overline{\mathrm{q}}}$ in the continuum limit and the results obtained with the Iwasaki and DBW2 actions at the largest $\beta$ at our disposal. For the Iwasaki action no appreciable difference in the results obtained with $r_{n}$ and $r_{\mathrm{I}}$ can be seen, while for the DBW2 action the discrepancy becomes large at small distances.

At large enough distances one obtains a good scaling in the coupling, and one does not observe scaling violation within the statistical errors. At small $r / r_{0}$ one sees deviations from the continuum limit, as one can observe in Fig. 9, where only the short distance region is considered. At $r \sim 0.4 r_{0}$ and $a \sim 0.09 \mathrm{fm}$, the deviations can be estimated to about $2 \%$ for the Iwasaki action; for the DBW2 action they amount to $4 \%$ if one uses $r_{n}$ to define the force, and reach even $40 \%$ by employing $r_{\mathrm{I}}$. This fact shows that the adoption of a tree level improved definition of the force does not guarantee success in reducing the lattice artefacts; in particular for RG actions, which are over-corrected at tree level, one should always check the scaling violations for different observables in order to obtain safe statements.

Note that for the DBW2 action the lattice artefacts in $T_{c} r_{0}$ are large when 


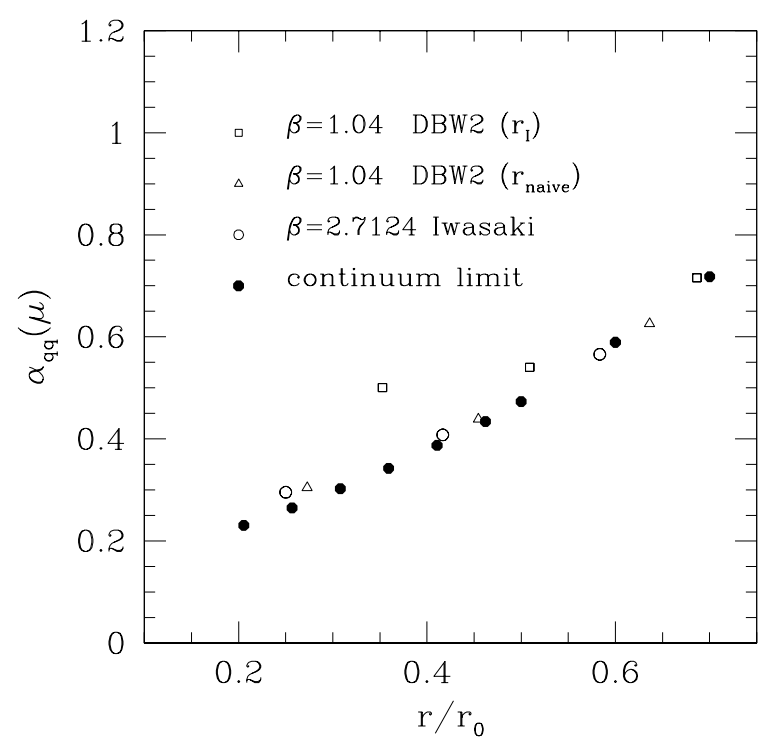

Figure 9: $\alpha_{\mathrm{q} \overline{\mathrm{q}}}$ at finite lattice spacing for Iwasaki and DBW2 action compared with the continuum results in the short distance region.

one uses $r_{n}$ to define the force. Thus there is not one definition of the force at finite $a$, which has reasonably small lattice artefacts for both $T_{c} r_{0}$ and $\alpha_{\mathrm{q} \overline{\mathrm{q}}}$.

\section{Glueball masses}

For the computation of the glueball masses we followed the method proposed by [12].

We decided to concentrate on the states $J^{P C}=0^{++}, 2^{++}$by measuring the masses in the irreducible representations $A_{1}^{++}, E^{++}$and $T_{2}^{++}$of the cubic group. $A_{1}$ corresponds to the $J=0$ state; in the continuum limit one expects that the $\mathrm{O}(3)$ symmetry is restored and hence the doublet $E$ and the triplet $T_{2}$ are degenerate and form together the $J=2$ quintuplet.

The $m_{0^{++}}$is particularly interesting for our purpose to investigate the lattice artefacts on RG actions since these turn out to be quite sizeable for the Wilson action.

We performed a test simulation using the standard Wilson action for $\beta=6.0$ on a $16^{4}$ lattice; here we measured all 22 loop shapes up to length 8 that can be build on the lattice (see [31] for the classification of the loops) and formed the wave functions corresponding to the representations $R=A_{1}^{++}, E^{++}, T_{2}^{++}$. Our 
timeslice observable at time $t$ is

$$
S_{n}^{R}(t)=\frac{L^{-3 / 2}}{K} \sum_{\vec{x}} \sum_{i=1}^{d_{n}} c_{n}^{i R} \operatorname{Re} W_{n}^{i}(\vec{x}, t), \quad n=1, \ldots, 22,
$$

where the coefficient $c_{n}^{i R}$ are taken from the literature [31,32], the sum over $i$ indicates the sum over all $d_{n}$ orientations of a given shape $n$ and $K$ is a suitable normalization constant.

Then we build the correlation matrices ${ }^{1}$

$$
C_{k l}^{R}(t)=\left\langle S_{k}^{R}(t) S_{l}^{R}(0)\right\rangle_{c}=\left\langle S_{k}^{R}(t) S_{l}^{R}(0)\right\rangle-\left\langle S_{k}^{R}(t)\right\rangle\left\langle S_{l}^{R}(0)\right\rangle .
$$

The indices $k, l$ assume $(22 \times M)$ values, where $M$ is the number of smearing levels. We adopted the same smearing procedure [22] that we used for the computation of the Wilson loops.

Starting from this large basis, we analyzed the signal/noise ratio of the different operators in order to eliminate those which introduce large noise and to reduce the correlation matrix to a set of well measurable operators.

We found general agreement in the classification of the "bad" operators with [12]. Our final choice for the operators to measure was then

$$
\begin{array}{lll}
A_{1}^{++} & : & \# 2, \# 5, \# 7, \# 8, \# 10, \# 12, \# 14 \\
E^{++} & : & \# 2, \# 5, \# 7, \# 10, \# 12, \# 14 \\
T_{2}^{++} & : & \# 7, \# 12, \# 14,
\end{array}
$$

where for $E^{++}$and $T_{2}^{++}$channels we took respectively 2 and 3 orthogonal projections for each shape. For each operator we then considered all $M$ smearing levels. We assumed that this choice is reasonable for each value of the lattice spacing and also for the Iwasaki and DBW2 actions and we stress that we did not perform a systematic study on the features of the several operators.

The simulation parameters for our new Monte Carlo simulations are listed in Table 6. Measurements were taken after a number of sweeps between 3 and 5 .

\subsection{Analysis details}

As already explained in sec. 4, due to the violation of physical positivity for the RG actions (see app.A), the variational method $[28,29]$ is mathematically not well founded, at least not at small time separations, where one would like to apply

\footnotetext{
${ }^{1}$ Notice that the vacuum subtraction is required only in the $A_{1}^{++}$channel, since it has the same quantum numbers as the vacuum.
} 
Iwasaki action

\begin{tabular}{cccc}
\hline$\beta$ & $L$ & $n_{l}$ & $n_{\text {meas }}$ \\
\hline 2.2423 & 10 & $2,4,6,8$ & 12000 \\
2.2879 & 12 & $2,4,6,8$ & 16000 \\
2.5206 & 16 & $3,6,9,12$ & 8000 \\
\hline \hline \multicolumn{4}{c}{ DBW2 action } \\
\hline$\beta$ & $L$ & $n_{l}$ & $n_{\text {meas }}$ \\
\hline 0.8342 & 12 & $2,4,6,8$ & 8000 \\
0.9636 & 16 & $3,6,9,12$ & 2500 \\
\hline
\end{tabular}

Table 6: Simulation parameters for the evaluation of the glueball masses for the Iwasaki and DBW2 actions.

it. The statistical errors are indeed drastically increasing with $t$ and already at $t=4 a$ the signal in the correlation function eq. (7.2) is lost in noise.

We decided to follow [12] by first solving the generalized eigenvalue problem

$$
C\left(t_{1}\right) v_{\alpha}\left(t_{1}, t_{0}\right)=\lambda_{\alpha}\left(t_{1}, t_{0}\right) C\left(t_{0}\right) v_{\alpha}\left(t_{1}, t_{0}\right),
$$

with $t_{0}=0, t_{1}=a$. Then we projected the correlation matrices to the space of eigenvectors corresponding to the $N$ eigenvalues which satisfy the condition ${ }^{2}$

$$
\lambda_{\beta}>\epsilon, \quad \beta=1, \ldots, N,
$$

where $\epsilon$ is an adjustable (small) parameter. So the reduced matrix is obtained by

$$
C_{i j}^{N}(t)=\left(v_{i}\left(t_{1}, t_{0}\right), C(t) v_{j}\left(t_{1}, t_{0}\right)\right), \quad i, j=1, \ldots, N,
$$

where the index $R$ for the representation in now omitted.

By choosing $\epsilon$ appropriately in eq. (7.7) one hopes to get rid of most of the unphysical modes caused by negative and very small eigenvalues.

Then one can apply the variational method to the reduced matrix

$$
C^{N}(t) w_{\beta}\left(t, t_{0}\right)=\lambda_{\beta}\left(t, t_{0}\right) C^{N}\left(t_{0}\right) w_{\beta}\left(t, t_{0}\right) .
$$

The effective glueball mass can be read off directly from the largest eigenvalue corresponding to the lowest energy

$$
m_{e f f}(t)=-\log \left(\frac{\lambda_{0}\left(t, t_{0}\right)}{\lambda_{0}\left(t-a, t_{0}\right)}\right) .
$$

\footnotetext{
${ }^{2}$ In [12] the direct eigenvalues and eigenvectors of $C\left(t_{0}\right)$ are considered, instead of the generalized one. We have tried both possibilities and found consistent results.
} 
or, alternatively one can project again $C^{N}$ to the subspace corresponding to the largest eigenvalue

$$
C^{1}(t)=\left(w_{1}, C^{N}(t) w_{1}\right)
$$

where $w_{1}=w_{1}\left(t_{0}+a, t_{0}\right)$; then one evaluates the glueball masses by

$$
m_{e f f}(t)=-\log \left(\frac{C^{1}(t)}{C_{1}(t-a)}\right) .
$$

We tested that these two different evaluation yield results which are compatible within the statistical errors.

We chose $\epsilon$ in eq. (7.7) so that the reduced matrix had dimension between 2 and 5.

We applied the same procedure also for $t_{0}=a, t_{1}=2 a$ in eq. (7.6), but due to the fact that the statistical fluctuations are already quite large one has to start from the beginning from a reduced number of operators in order to be able to solve the generalized eigenvalue problem.

We decided to extract the glueball masses by taking the effective mass at $t=3 a$ for the small $\beta$ regime and $t=4 a$ for the large value of the coupling at our disposal. For the RG actions we performed numerical simulations up to $L=16$, with a minimum lattice spacing $a \sim 0.1 \mathrm{fm}$, which is quite large and hence we will not be able to perform a continuum extrapolation of the results. On the other hand, this is the regime of lattice spacings that have been used for simulations with dynamical fermions and for this reason it is desirable to obtain informations about the discretization errors in this region.

The figures 10,11 show the effective masses in the $A_{1}^{++}$channel computed by using eq. (7.10) (filled squares) and eq. (7.12) (empty squares) for the Iwasaki and the DBW2 action, with $t_{0}=0$. For the largest value of $\beta$, we show also the results obtained with $t_{0}=a$ (filled triangles). As for the potential, one notices also in this case the presence of negative contributions in the correlation functions due to unphysical states; the situation here is somehow more drastic since one has to discard for this reason the small $t$ region but on the other hand the errors increase very rapidly and one is forced to extract the glueball mass at $t=(3-4) a$. The results can then be affected by systematic errors that can not be easily estimated. In the plots we have indicated with the dotted lines the range in which we decided to take the mass. The statistical errors were evaluated by using a jackknife procedure.

Concerning the determination of $m_{2^{++}}$we observed that the signal for the $E^{++}$ channel is usually worse than for the $T_{2}^{++}$and the errors on the effective masses are very large already at $t=3 a$. For this reason we decided to use $m_{T_{2}^{++}}$as estimate of $m_{2^{++}}$at finite lattice spacing. 
Figure 12 shows the effective masses for the $T_{2}^{++}$channel. The determination of the masses is more problematic that the $A_{1}^{++}$case; in particular for the largest coupling one can observe that at $t=4 a$ the errors are too large to have a significant measurement of the mass. We decided hence to extract the mass at $t=3 a$, taking care that this value is compatible with the one at $t=4 a$ within the statistical errors.

\section{$7.2 \quad$ Results}

In Table 8 the results for the masses in lattice units are reported. For the smallest $\beta$ for the Iwasaki action, it was not possible to obtain a reliable evaluation of the mass in the $T_{2}^{++}$channel.

The renormalized quantities $r_{0} m_{G}$ are reported in tables 9 and 10; due to the fact that the errors on $a m_{G}$ are quite large (between $8 \%$ and $9 \%$ for $A_{1}^{++}$and between $10 \%$ and $20 \%$ for $T_{2}^{++}$), the difference which arises by choosing $r_{I}$ or $r_{n}$ is in this case much smaller than the total uncertainty; we have reported however both results in the table. For $\beta=2.2423$ we had no direct measurement of $r_{0} / a$ and we made use of the interpolation formula eq. (4.9).

Figure 13 shows the results for $r_{0} m_{0^{++}}$as function of $\left(a / r_{0}\right)^{2}$, displaying only the results obtained with $r_{n}$. For the comparison we included the results for FP action [12] and several calculations performed with the Wilson action [33,34,35].

The continuum values avaliable in the literature are listed in Table 7 and have been taken from [12] for the FP action and from [36] for the Wilson action, where the results of $[33,34,35]$ have been expressed in units of $r_{0}$.

The interpretation of our results is not very clear, also due to the large errors.

In any case, we want to stress that our determination can be seen at least as an upper limit for $m_{0^{++}}$and $m_{2^{++}}$. We expect that at the values of $t / a$ at which we extracted the masses, the effects of positivity violations have already disappeared; this assumption is justified by our study based on perturbation theory and our estimation of $t_{\min }$ eq. (A.9).

For this reason we believe that possible systematic uncertainties on the glueball masses could only be due to the presence of excited states and hence could affect our measurement only in such a way that the real values of $m_{0^{++}}, m_{2^{++}}$are lower with respect to our determination.

At lattice spacings $a \sim 0.15 \mathrm{fm}$ we notice a improvement of the RG actions with respect to the Wilson action; comparing with the continuum limit we find no significant discrepancy both for DBW2 and Iwasaki action, while for the Wilson action one finds $30-40 \%$ deviation.

At lattice spacing $a \sim 0.1 \mathrm{fm}$ we find on the other hand large lattice artefacts for 

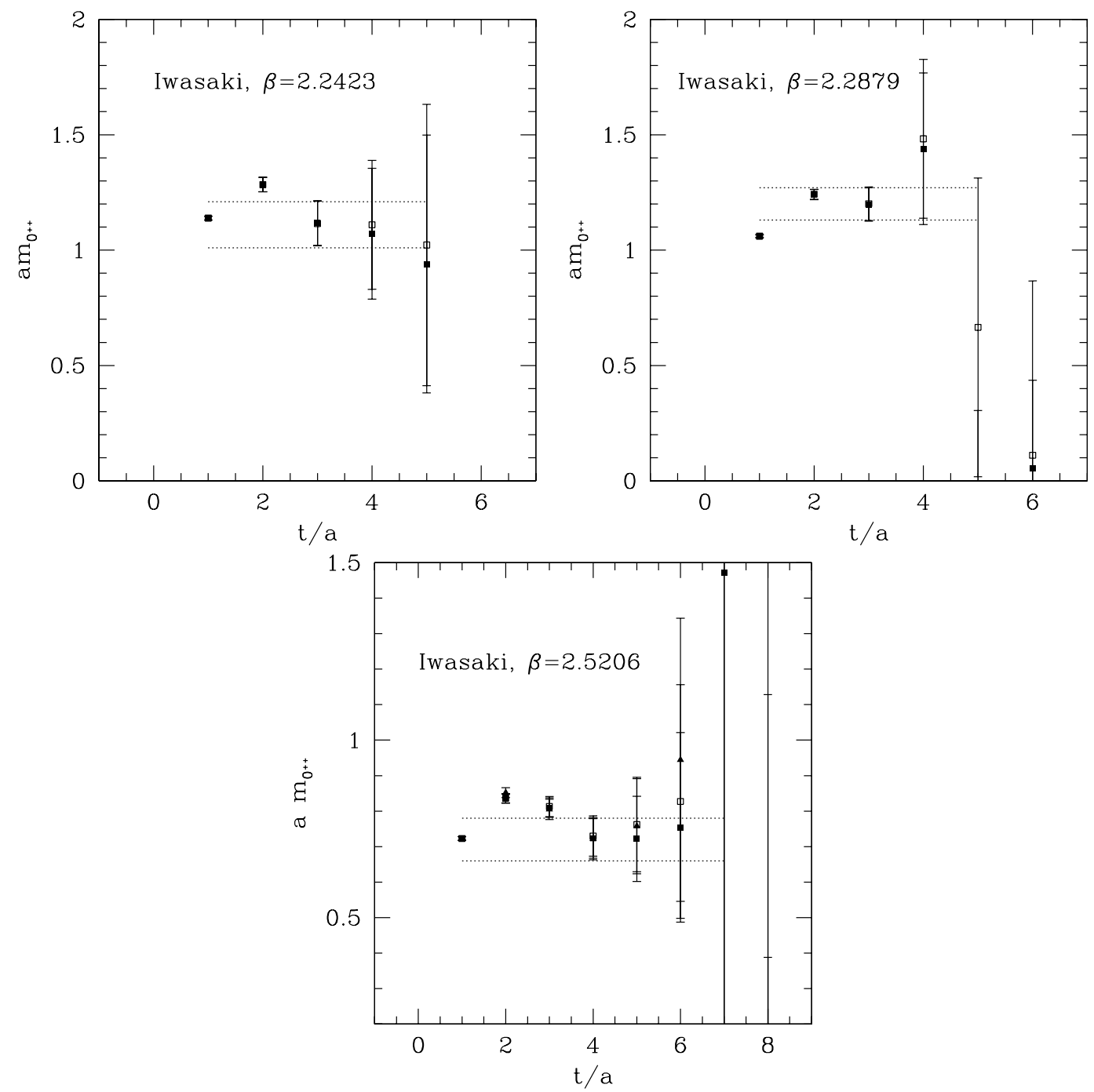

Figure 10: The effective masses for the $A_{1}^{++}$channel, evaluated with Iwasaki action, at different lattice spacings. The filled and empty squares corresponds to respectively to eq. (7.10) and eq. (7.12) with $t_{0}=0$. For $\beta=2.5206$, the filled triangles correspond to eq. (7.10) with $t_{0}=a$. 

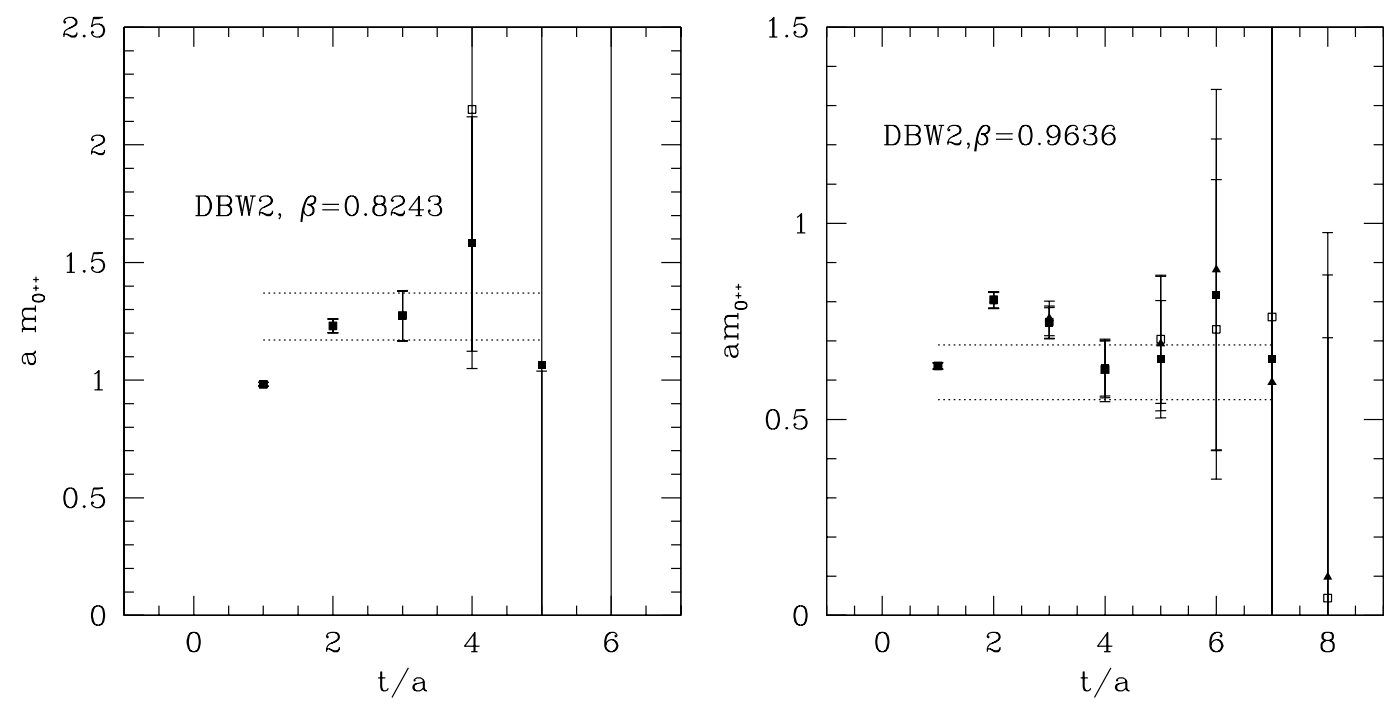

Figure 11: The effective masses for the $A_{1}^{++}$channel, evaluated with DBW2 action, at different lattice spacings. The filled and empty squares corresponds to respectively to eq. (7.10) and eq. (7.12) with $t_{0}=0$. For $\beta=0.9636$, the filled triangles correspond to eq. (7.10) with $t_{0}=a$.

RG actions: the result obtained with the Iwasaki action is compatible with the one calculated through the plaquette action at the same lattice spacing, while for the DBW2 action it is even further away from the continuum limit.

If one considers our measurement as upper limit, one could conclude that the RG improved actions are not able to cure the problem of large lattice artefacts for the $0^{++}$glueball mass. At very small lattice spacings one expects that the dominant lattice artefacts are of order $a^{2}$; for the Wilson action this is indeed well confirmed by the numerical results in the range $a \lesssim 0.17 \mathrm{fm}$, as one can see in Fig. 13.

For alternative actions there is no reason a priori to observe the same behavior: while at small lattice spacings the $\mathrm{O}\left(a^{2}\right)$ should in any case be the dominant one, at larger $a$ it is possible that lattice artefacts are governed by higher orders $\left(\mathrm{O}\left(a^{4}\right)\right.$ and higher).

We have indeed already observed deviations from $\mathrm{O}\left(a^{2}\right)$ behavior in RG actions for the quantity $T_{c} r_{0}$ and even already for the force computed at tree level in the previous sections.

In Fig. 14 we report our results for $r_{0} m_{2^{++}}$; for this particular observable the calculation performed with the Wilson action do not show significant lattice artefacts.

At our smallest lattice spacing we do not observe a deviation from the results obtained with the Wilson action; one has however to notice that our errors are too large to make any conclusive statement.

For our largest lattice spacing the results with the Wilson action are not available 

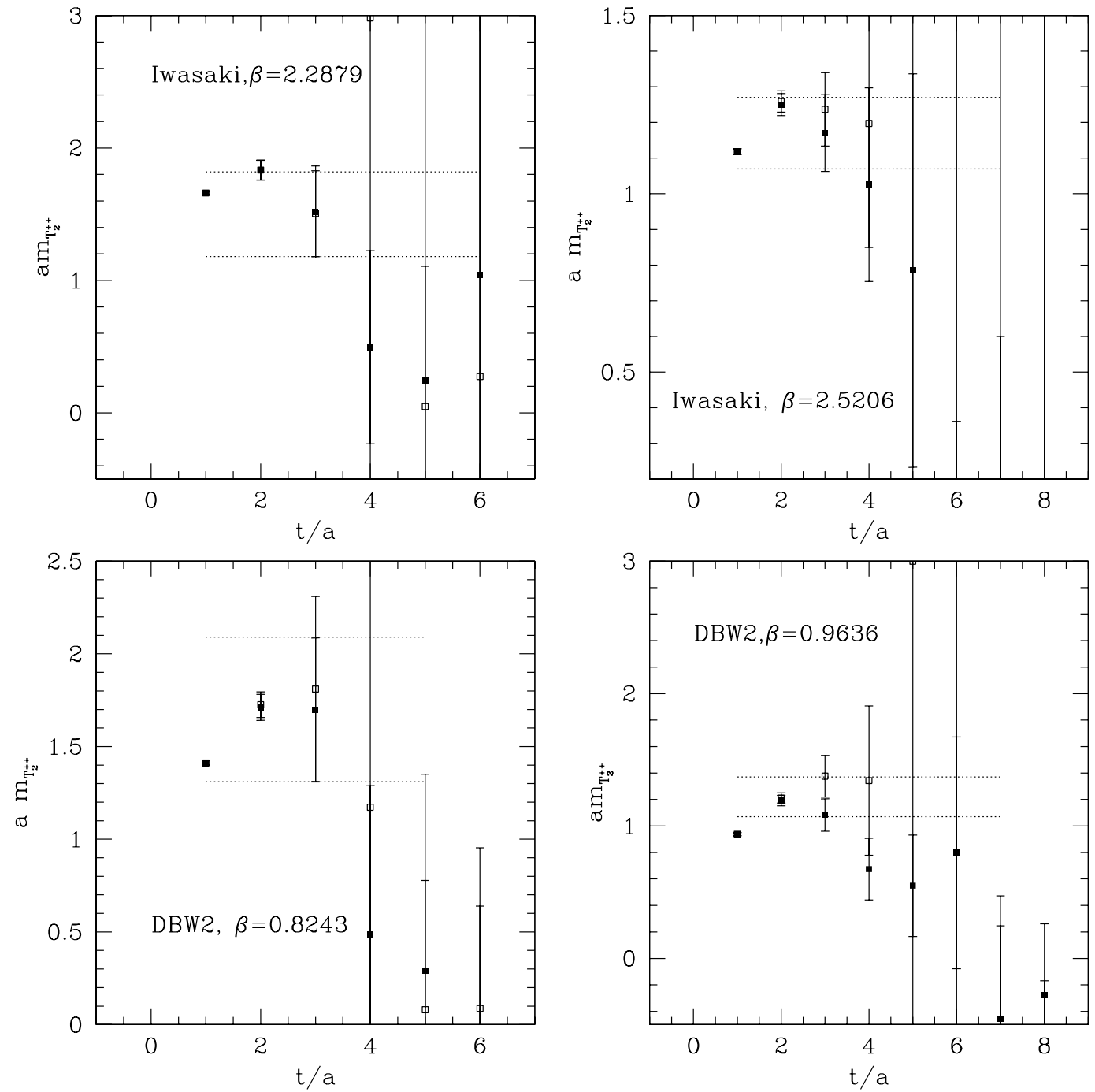

Figure 12: The effective masses for the $T_{2}^{++}$channel, evaluated with Iwasaki and DBW2 action, at different lattice spacings. The filled and empty squares corresponds to respectively to eq. (7.10) and eq. (7.12) with $t_{0}=0$. 


\begin{tabular}{ccc}
\hline Collab. & $r_{0} m_{0^{++}}$ & $r_{0} m_{2^{++}}$ \\
\hline M \& P [37] & $4.21(11)(4)$ & $5.85(2)(6)$ \\
GF11 [34] & $4.33(10)$ & $6.04(18)$ \\
Teper [19] & $4.35(11)$ & $6.18(21)$ \\
UKQCD [35] & $4.05(16)$ & $5.84(18)$ \\
FP [12] & $4.12(21)$ & {$[5.96(24)]$} \\
\hline
\end{tabular}

Table 7: Continuum extrapolations of the two lowest glueball masses in units of $r_{0}$. For the FP action, the $2^{++}$value is not extrapolated to the continuum but denotes the mass obtained at a lattice spacing $a=0.10 \mathrm{fm}$.

\begin{tabular}{ccc}
\multicolumn{4}{c}{ Iwasaki action } \\
\hline$\beta$ & $a m_{A_{1}^{++}}$ & $a m_{T_{2}^{++}}$ \\
\hline 2.2423 & $1.11(10)$ \\
2.2879 & $1.20(7)$ & $1.50(32)$ \\
2.5206 & $0.72(6)$ & $1.17(10)$ \\
\hline \multicolumn{3}{c}{ DBW2 action } \\
\hline \multicolumn{3}{c}{$a m_{A_{1}^{++}} a m_{T_{2}^{++}}$} \\
\hline 0.8243 & $1.27(10)$ & $1.70(39)$ \\
0.9636 & $0.62(7)$ & $1.22(15)$ \\
\hline
\end{tabular}

Table 8: Glueball masses in lattice units, Iwasaki and DBW2 action.

(there are only know results with anisotropic lattices [37]) and it is indeed difficult to have a reliable estimation of the mass also in our case: we decided to show however our results, even with the very large error bars.

From our computation one can not deduce if for $r_{0} m_{2^{++}}$the RG actions show significative discretization errors and further investigations are needed to clarify the issue.

In particular, the exponential error reduction proposed by Lüscher and Weisz in [38] has been already tested for the evaluation of the $0^{++}$and $2^{++}$masses in [39] yielding promising results. We expect that the implementation of this algorithm for RG actions could help in reducing the errors for the correlation functions at quite large $t$ and hence to have more reliable estimations of the glueball masses. 
Iwasaki action

\begin{tabular}{ccccc}
\hline$\beta$ & $r_{0} m_{A_{1}^{++}}\left(r_{n}\right)$ & $r_{0} m_{T_{2}^{++}}\left(r_{n}\right)$ & $r_{0} m_{A_{1}^{++}}\left(r_{\mathrm{I}}\right)$ & $r_{0} m_{T_{2}^{++}}\left(r_{\mathrm{I}}\right)$ \\
\hline 2.2423 & $3.08(28)$ & & $3.07(28)$ & \\
2.2879 & $3.63(21)$ & $4.54(97)$ & $3.63(21)$ & $4.54(97)$ \\
2.5206 & $3.26(27)$ & $5.31(45)$ & $3.25(27)$ & $5.28(45)$ \\
\hline
\end{tabular}

Table 9: Results for $r_{0} m_{G}$ for the channels $A_{1}^{++}$and $T_{2}^{++}$, using the Iwasaki action.

\begin{tabular}{ccccc}
\multicolumn{5}{c}{ DBW2 action } \\
\hline$\beta$ & $r_{0} m_{A_{1}^{++}}\left(r_{n}\right)$ & $r_{0} m_{T_{2}^{++}}\left(r_{n}\right)$ & $r_{0} m_{A_{1}^{++}}\left(r_{\mathrm{I}}\right)$ & $r_{0} m_{T_{2}^{++}}\left(r_{\mathrm{I}}\right)$ \\
\hline 0.8243 & $3.97(31)$ & $5.3(1.2)$ & $3.86(30)$ & $5.2(1.2)$ \\
0.9636 & $2.86(32)$ & $5.62(69)$ & $2.82(32)$ & $5.56(68)$ \\
\hline
\end{tabular}

Table 10: Results for $r_{0} m_{G}$ for the channels $A_{1}^{++}$and $T_{2}^{++}$, using the DBW2 action.

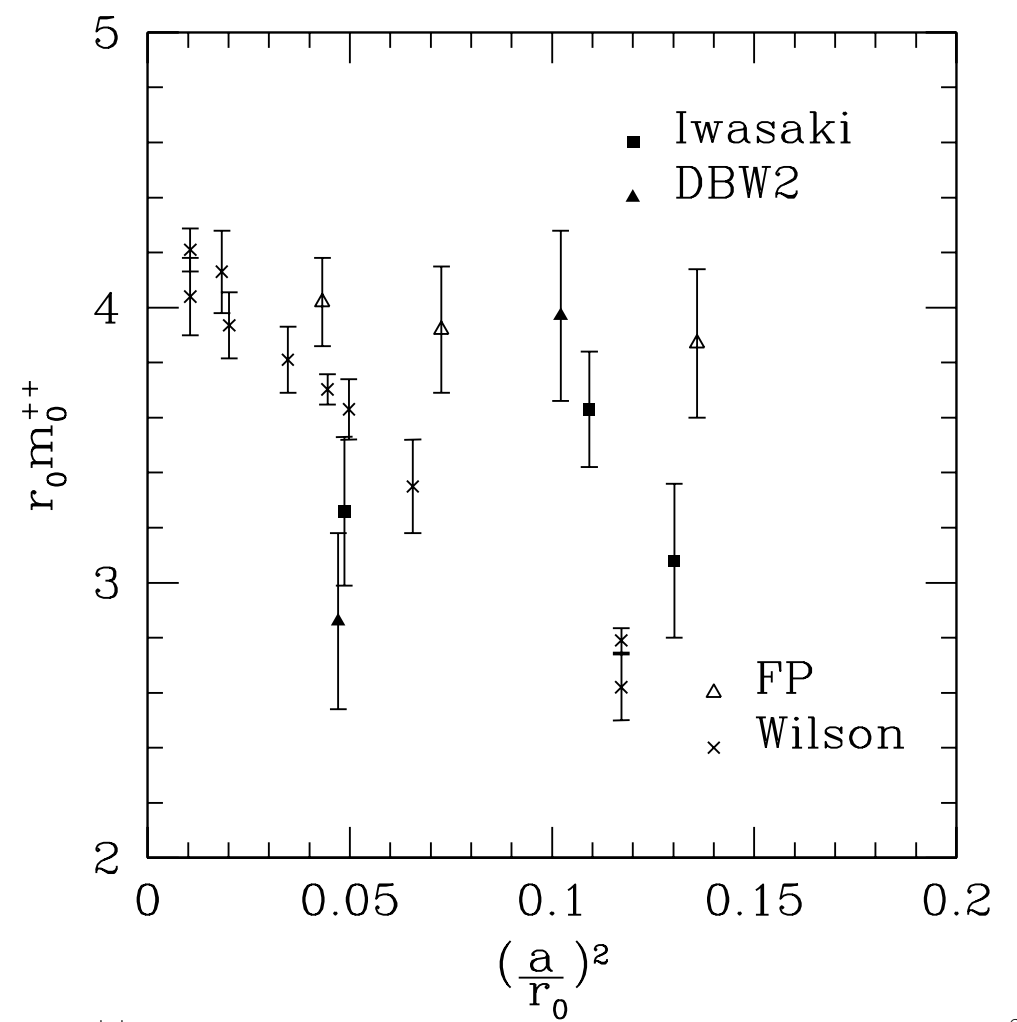

Figure 13: The $0^{++}$glueball mass normalized with $r_{0}$ as function of $\left(a / r_{0}\right)^{2}$ for different actions. 


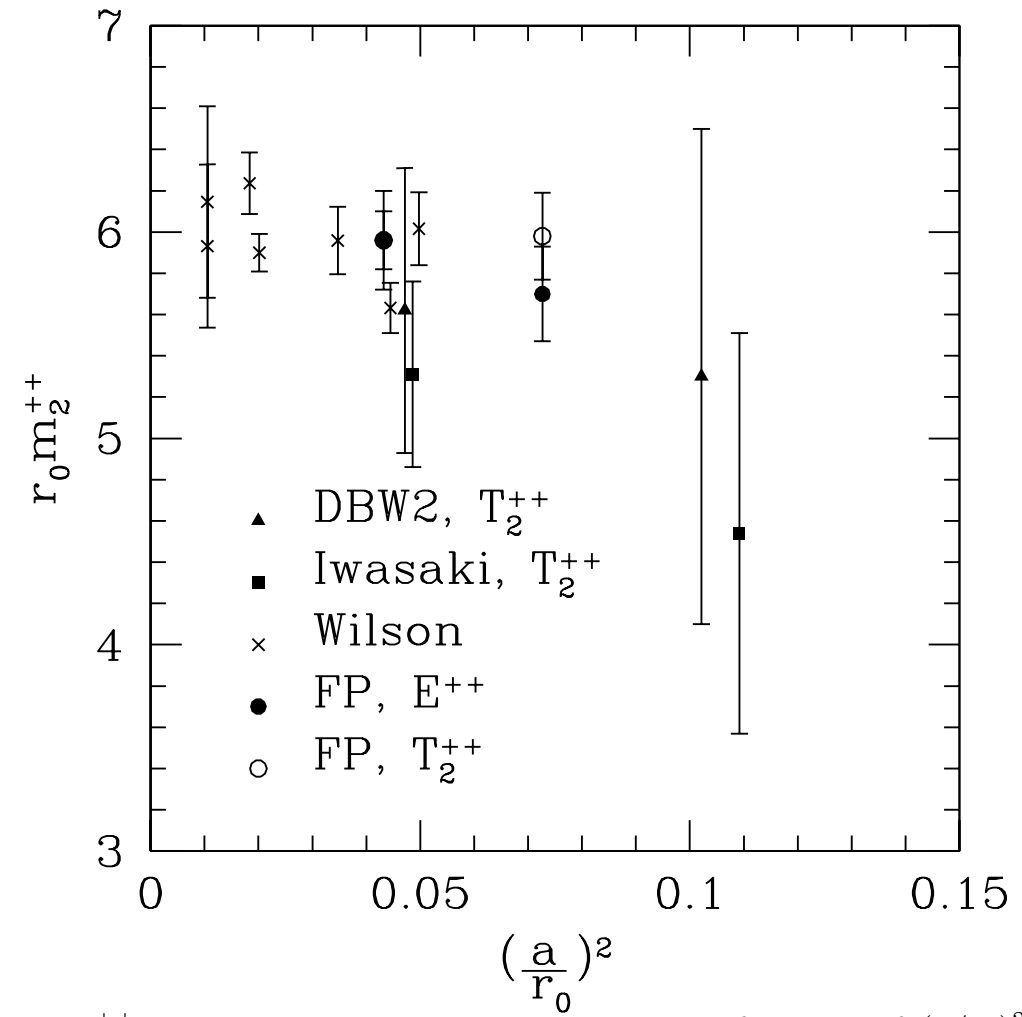

Figure 14: The $2^{++}$glueball mass normalized with $r_{0}$ as function of $\left(a / r_{0}\right)^{2}$ for different actions. 


\section{Discussion}

The adoption of alternative gauge actions with the purpose to reduce the lattice artefacts and/or to improve chiral properties in QCD simulations must be accompanied with an accurate study of their properties. In particular, universality and scaling behavior must be tested on a large set of observables.

We have chosen $r_{0}$, the deconfining temperature and the glueball masses $m_{0^{++}}$, $m_{2^{++}}$to perform this test for the Iwasaki and DBW2 actions, which contain the same operators with different coefficients.

We computed the scale $r_{0}$ through the force between static quarks at several values of $\beta$ corresponding to the critical couplings for deconfinement and finally built the renormalized quantity $T_{c} r_{0}$. By approaching the continuum limit we verified the universality between Wilson and RG action, which had been doubted in previous calculations where the string tension $\sigma$ was used to set the scale instead of $r_{0}$. Our new computation confirms that $r_{0}$ is a more appropriate quantity, since the systematic uncertainties are reduced with respect to the string tension.

From the point of view of the scaling behavior, for this particular quantity the Iwasaki action is able to reduce the lattice artefacts. For the DBW2 action, on the other hand, we observe large lattice artefacts at $a \sim \ldots .$. While they can be made small in $r_{0} T_{c}$ by choosing a $r_{\mathrm{I}}$ in the definition of the force, this choice leeds to large $a$-effects in $\alpha_{\mathrm{q}}$. The coefficient $c_{1}$ appears to be too far away from its tree-level value. By investigating the force at tree level we pointed out that RG actions are "over-corrected", in the sense that they can introduce even larger lattice artefacts than the Wilson action. This suggests that the improvement may work only in specific coupling ranges; moreover, it can happen that the $\mathrm{O}\left(a^{2}\right)$ scaling violation is dominant only at very small lattice spacings, and this feature requests a particular care in performing the continuum extrapolation.

Another important fact that we stressed is the violation of physical positivity which occur in RG actions, and more generally in actions that contain other terms apart from the plaquette. We clearly observed the presence of unphysical states in the evaluation of the static quark potential from Wilson loop correlation functions.

The main problem connected with this fact is the failure in the mathematical assumptions which allow to apply the variational method. The method can be safely applied only if one manages to get rid of the unphysical modes by going at sufficiently large time separations - but here the statistical errors increase dramatically - or by finding a reliable procedure to project them out from the correlation matrix.

We followed [27] and estimated in perturbation theory at which time separation 
these lattice artefacts are expected to disappear and this indicative study is confirmed by our numerical evaluation of the static potential.

We stress that we did not perform a systematic study on the efficiency of the smearing procedure for RG actions and we applied the same criteria adopted for the Wilson action at analogous lattice spacings. The presence of states with negative norm can spoil the search for an "optimal" smearing since it can mask the presence of excited states at small time separations, and a reasonable criterium must be found.

In a second part of the work we performed numerical simulations to evaluate $m_{0^{++}}$ and $m_{2^{+}}$at several lattice spacings for $\mathrm{RG}$ actions. The main motivation was that the $0^{++}$glueball show large scaling deviations and hence is an ideal observatory to make a comparison with alternative actions.

Here the violation of positivity constitutes an even stronger problem, since for these observables the signal is lost in noise already at small time separations.

Due to these difficulties, our estimations of glueball masses are affected by large errors and hence not suitable to draw a definitive conclusion about the lattice artefacts; a possible solution would be to apply specific algorithms for the variance reduction in order to extend the precision on the effective masses to larger values of $t$, where the unphysical states are supposed to be absent.

The fact that for the Wilson action the lattice artefacts for $m_{0^{++}}$are large and the glueball mass in lattice units becomes small is usually interpreted as the "influence" of the endpoint of the first order phase transition which for SU(3) plaquette action is located in the fundamental-adjoint coupling plane at $\left(\beta_{f}, \beta_{a}\right)=$ $(4.00(7), 2.06(8))[40]$.

This issue turns out to be very important for the next simulation with dynamical fermions; in particular, in $O(a)$ improved Wilson fermions it is believed that the clover term produces an adjoint term in the gauge action with positive coupling which could be responsible for the observed first order phase transition at zero-temperature in the $N_{f}=3$ simulations [41]. The JLQCD investigations indicate that this transition is a lattice artefact restricted to strong coupling regions $(\beta \lesssim 5.0)$ and it disappears if one adopts other gauge actions, like Iwasaki or tadpole-improved Symanzik action, or alternatively if one uses the unimproved Wilson fermionic action with the plaquette gauge action.

By considering our estimation as an upper limit for the glueball masses, it seems that RG actions do not lead to a sensible reduction of the lattice artefacts for $m_{0^{++}}$, and hence the scenario remains unclear. A possible alternative to avoid the problem would be to adopt the usual plaquette action with a negative adjoint term, and studies on the scaling behavior for this kind of actions are ongoing. 


\section{Aknowledgment}

I would like to thank Rainer Sommer, Peter Weisz, Martin Lüscher, Urs Wenger, Francesco Knechtli and Stephan Dürr for useful discussions and suggestions. Part of the computation was performed on a CRAY-T3e at Forschungszentrum Jülich; we thank NIC for providing CPU resources and assistance.

\section{A Positivity violation in improved gauge actions}

In [27] it has been pointed out that for lattice gauge actions containing general classes of loops it is still possible to construct a transfer matrix $\mathcal{T}$. In particular, restricting to actions of kind eq. $(2.1)$ with plaquettes and planar $(1 \times 2)$ loops, the transfer matrix can be defined as transition amplitude between fields configurations defined on two consecutive pairs of equal time hyperplanes. But, unlike the Wilson case, one finds that $\mathcal{T}$ is no longer hermitean, and its spectral values $\lambda$ occur in pairs of complex conjugated numbers.

Moreover, one can show that the spectrum $\sigma(\mathcal{T})$ contains a real, positive, nondegenerate eigenvalue $\lambda_{0}$ such that

$$
\lambda_{0}>|\lambda|, \quad \forall \lambda \in \sigma(\mathbb{T}), \quad \lambda \neq \lambda_{0} .
$$

The corresponding eigenfunction may be interpreted as ground state wave function.

The violation of physical positivity forbids the definition of an Hamiltonian operator; from the point of view of the spectral decomposition of connected two-point functions, one still observes expontential decay at large time-separations, but contributions with negative weight will appear, as remnant of the positivity violation. Nevertheless, the violation of positivity is a lattice artefact and one expects that physical positivity is recovered in the continuum limit; one should be able to define a subspace of states with small energy with respect to the cut-off where the non-hermiticity of the trasfer matrix can be eliminated.

This corresponds to the fact that for large enough time-separations, the negative contributions to the spectral decomposition of two-point functions should disappear.

Following [27], one can argue that there exists a $0<\epsilon<1$ such that independently of the cutoff the following properties hold:

(i) all spectral values $\lambda \in \sigma(\mathbb{T})$ with $|\lambda| \geq \epsilon \lambda_{0}$ are real and positive;

(ii) one can define a physical Hilbert space $\mathcal{H}_{\text {phys }} \in \mathcal{H}$ and a new scalar product such that $(\Psi, \Psi)_{\text {new }}>0, \quad \forall \Psi \in \mathcal{H}_{\text {phys }}, \quad \Psi \neq 0$.

The existence of such $\epsilon$ has not been rigorously proved, but it is supported for 
example by perturbative calculations. In the next section we will estimate $\epsilon$ by determining the location of unphysical poles in the free propagator.

\section{A.1 Unphysical poles in the propagator}

In [9] the propagator $D_{\mu \nu}$ associated to the action eq. (2.1) is evaluated to lowest order in $g_{0}$ with covariant gauge fixing; it is defined by

$$
\left\langle A_{\mu}^{i}(x) A_{\nu}^{j}(y)\right\rangle=\delta^{i j} \int_{k} e^{i k(x-y)} e^{i\left(k_{\mu}-k_{\nu}\right) / 2} D_{\mu \nu}(k),
$$

where $A_{\mu}(x)$ is related to the link variables by

$$
U(x, \mu)=e^{-A_{\mu}(x)}
$$

and the momenta integration corresponds to

$$
\int_{k}=\prod_{\mu=0}^{3} \int_{-\pi}^{\pi} \frac{d k_{\mu}}{2 \pi}
$$

We are now referring to the case $L=\infty$, so that the momenta take continuous values in the Brillouin zone.

Eq. (A.2) can be rewritten in the form

$$
D_{\nu \tau}(k)=\left(\hat{k}^{2}\right)^{-2}\left[\alpha \hat{k}_{\nu} \hat{k}_{\tau}+\sum_{\sigma}\left(\hat{k}_{\sigma} \delta_{\tau \nu}-\hat{k}_{\tau} \delta_{\sigma \nu}\right) A_{\tau \sigma}(k) \hat{k}_{\sigma}\right],
$$

where $\alpha$ is the gauge parameter, $\hat{k}_{\mu}=2 \sin \left(k_{\mu} / 2\right)$ and $A_{\mu \nu}$ is independent of $\alpha$ and has the general form

$$
A_{\mu \nu}=\frac{f(\hat{k})}{D}
$$

with

$$
D=\sum_{\mu} \hat{k}_{\mu}^{4} \prod_{\nu \neq \mu} q_{\mu \nu}+\sum_{\mu>\nu, \rho>\tau,\{\rho, \tau\} \cup\{\mu, \nu\}=\emptyset} \hat{k}_{\mu}^{2} \hat{k}_{\nu}^{2} q_{\mu \nu}\left(q_{\mu \rho} q_{\nu \tau}+q_{\mu \tau} q_{\nu \rho}\right) .
$$

The explicit form of $f(\hat{k})$ can be found in [9] and will not reported here. The denominator of eq. (A.3) is then given by

$$
\begin{gathered}
\Delta=D\left(\hat{k}^{2}\right)^{2}= \\
\left(\hat{k}^{2}\right)^{2}\left(\sum_{\mu} \hat{k}_{\mu}^{4} \prod_{\nu \neq \mu} q_{\mu \nu}+\sum_{\mu>\nu, \rho>\tau,\{\rho, \tau\} \cup\{\mu, \nu\}=\emptyset} \hat{k}_{\mu}^{2} \hat{k}_{\nu}^{2} q_{\mu \nu}\left(q_{\mu \rho} q_{\nu \tau}+q_{\mu \tau} q_{\nu \rho}\right)\right),
\end{gathered}
$$


where $q_{\mu \nu}$ for our specific form of the action eq. (2.1) takes the form

$$
q_{\mu \nu}=\left(1-\delta_{\mu \nu}\right)\left[1-c_{1}\left(\hat{k}_{\mu}^{2}+\hat{k}_{\nu}^{2}\right)\right] .
$$

Then eq. (A.6) becomes

$$
\begin{gathered}
\Delta=\left(\hat{k}^{2}-c_{1} \sum_{\mu} \hat{k}_{\mu}^{4}\right)\left[\hat{k}^{2}-c_{1}\left(\left(\hat{k}^{2}\right)^{2}+\sum_{\mu} \hat{k}_{\mu}^{4}\right)+\right. \\
\left.\frac{1}{2} c_{1}^{2}\left(\left(\hat{k}^{2}\right)^{3}+2 \sum_{\mu} \hat{k}_{\mu}^{6}-\hat{k}^{2} \sum_{\mu} \hat{k}_{\mu}^{4}\right)\right]-4 c_{1}^{3} \sum_{\mu} \hat{k}_{\mu}^{4} \prod_{\nu \neq \mu} \hat{k}_{\nu}^{2} .
\end{gathered}
$$

In order to search for the poles of the propagator, one substitutes

$$
k=\left(\hat{k}_{1}, \hat{k}_{2}, \hat{k}_{3}, i w\right)
$$

and looks for solutions of the equation $\Delta=0$ scanning the whole Brillouin zone. In general for $c_{1} \neq 0$ one expects complex conjugated solutions

$$
w=\mathfrak{R e}(w) \pm i \mathfrak{I m}(w)=w\left(\hat{k}_{1}, \hat{k}_{2}, \hat{k}_{3}\right) .
$$

Numerical investigations showed that the condition $\mathfrak{I m}(w)=0$ (for all solutions at a given momentum) defines the equation $f\left(\hat{k}_{1}, \hat{k}_{2}, \hat{k}_{3}\right)=0$ which represents a compact 3-dimensional object. In Fig. 15 the 2-dimensional intersection with the plane $\hat{k}_{3}=0$ is plotted for several values of $c_{1}$ corresponding to different actions. For our numerical studies, we discretized the Brillouin zone in finite intervals that could be made arbitrarily small.

In the region enclosed by the curve one has $\mathfrak{I m}(w)=0$, while outside $\mathfrak{I m}(w) \neq$ 0 .

The Fig. 16 represents the distribution of the poles; by scanning the Brillouin zone and varying the intervals of the momenta, one reaches the conclusion that there exists a maximal value of $\mathfrak{R e}(w)$, which is related to $c_{1}$ by

$$
w_{\text {max }}=2 \operatorname{arcsinh}\left(\frac{1}{2 \sqrt{-2 c_{1}}}\right)= \begin{cases}2.063 & \text { Symanzik, tree level } \\ 1.162 & \text { Iwasaki } \\ 0.588 & \text { DBW2 }\end{cases}
$$

such that for $w<w_{\max }$ the imaginary part $\mathfrak{I m}(w)$ vanishes.

The value of $w_{\max }$ yields an estimate of $\epsilon$ defined above through the simple relation

$$
\epsilon=e^{-w_{\max }}= \begin{cases}0.127 & \text { Symanzik, tree level } \\ 0.313 & \text { Iwasaki } \\ 0.555 & \text { DBW2 }\end{cases}
$$




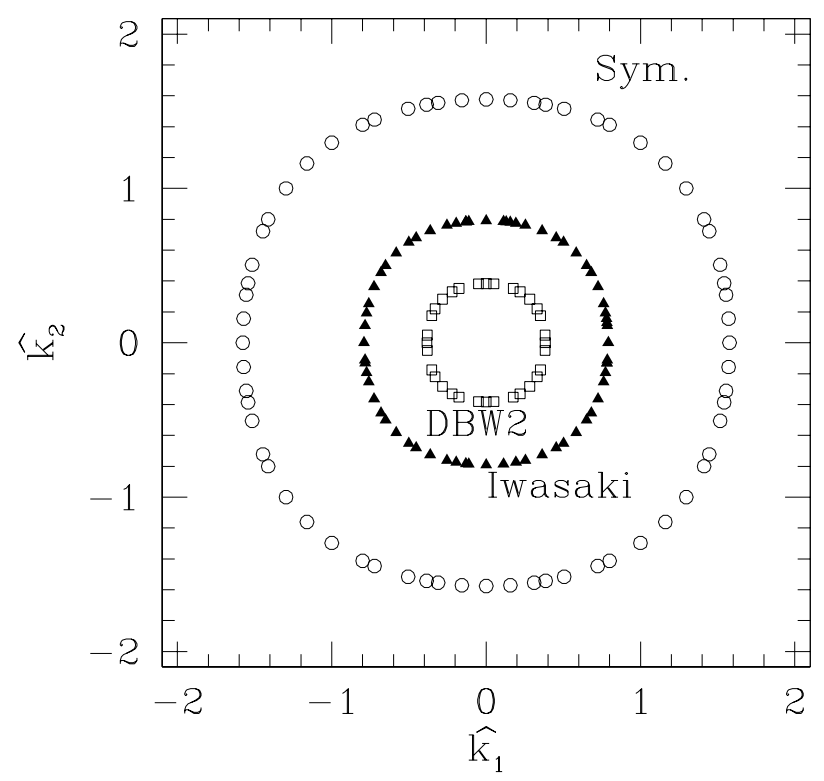

Figure 15: The curves defined by the condition $\mathfrak{I m}(w)=0$ in the $\hat{k}_{3}=0$ plane. Inside the curve $\mathfrak{I m}(w)=0$, while outside $\mathfrak{I m}(w) \neq 0$.

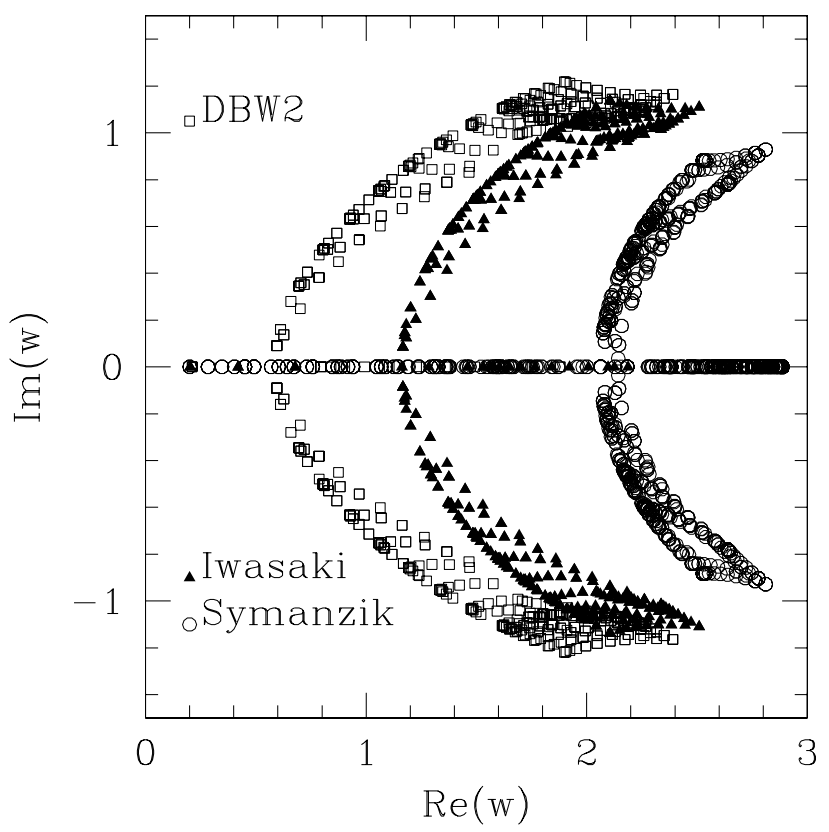

Figure 16: Distribution of the poles for different actions, scanning the Brillouin zone. 
Evaluating observables from exponential decays of correlation functions, one expects the presence of unphysical states unless $t \gg t_{m i n}$, where $t_{\min }$ in lattice units is given by

$$
t_{\text {min }}=\frac{1}{w_{\max }}= \begin{cases}0.484 & \text { Symanzik, tree level } \\ 0.860 & \text { Iwasaki } \\ 1.702 & \text { DBW2 }\end{cases}
$$

One has to remember that our estimation of the unphysical poles has been performed in perturbation theory and hence has to be considered as an indicative evaluation.

\section{B Numerical results}

The tables 11 and 12 report the data for the potential and the force evaluated at finite lattice spacing with Iwasaki and DBW2 actions. $r_{\mathrm{I}}$ defines the treelevel improved force and is defined in eq. (4.7). For the potential only the naive definition is reported. 
Table 11: The potential and the force in lattice units for the Iwasaki action.

\begin{tabular}{ccccc}
\hline$\beta$ & $r_{I} / a$ & $a^{2} F\left(r_{I}\right)$ & $r / a$ & $a V(r)$ \\
\hline 2.1551 & 1.4858 & $0.4059(21)$ & 2 & $0.9519(20)$ \\
& 2.4626 & $0.2965(75)$ & 3 & $1.2487(61)$ \\
& 3.5663 & $0.2585(98)$ & 4 & $1.515(12)$ \\
& 4.6059 & $0.249(29)$ & 5 & $1.783(12)$ \\
\hline 2.2879 & 1.4858 & $0.6055(29)$ & 2 & $0.7730(12$ \\
& 2.4626 & $0.19796(76)$ & 3 & $0.9707(33)$ \\
& 3.5663 & $0.1689(70)$ & 4 & $1.1418(41)$ \\
& 4.6059 & $0.1622(64)$ & 5 & $1.3047(95)$ \\
& 5.6082 & $0.155(10)$ & 6 & $1.460(16)$ \\
& 6.6036 & $0.148(16)$ & 7 & $1.608(33)$ \\
\hline 2.5208 & 1.4858 & $0.21301(25)$ & 2 & $0.60342(29)$ \\
& 2.4626 & $0.11734(39)$ & 3 & $0.72099(46)$ \\
& 3.5663 & $0.09011(70)$ & 4 & $0.8107(14)$ \\
& 4.6059 & $0.0803(10)$ & 5 & $0.8902(20)$ \\
& 5.6082 & $0.07575(61)$ & 6 & $0.9658(36)$ \\
& 6.6036 & $0.0744(32)$ & 7 & $1.0378(37)$ \\
& 7.5977 & $0.0711(15)$ & 8 & $1.1098(74)$ \\
\hline 2.7124 & 1.4858 & $0.17512(12)$ & 2 & $0.52165(14)$ \\
& 2.4626 & $0.08691(14)$ & 3 & $0.60856(20)$ \\
& 3.5663 & $0.06138(53)$ & 4 & $0.66994(56)$ \\
& 4.6059 & $0.05194(81)$ & 5 & $0.7219(13)$ \\
& 5.6082 & $0.04693(85)$ & 6 & $0.7688(20)$ \\
& 6.6036 & $0.0451(23)$ & 7 & $0.8139(11)$ \\
& 7.5977 & $0.0425(10)$ & 8 & $0.8564(10)$ \\
& 8.5915 & $0.0420(15)$ & 9 & $0.8984(23)$ \\
& 9.5854 & $0.0414(19)$ & 10 & $0.9398(25)$ \\
\hline
\end{tabular}


Table 12: The potential and the force in lattice units for the DBW2 action.

\begin{tabular}{ccccc}
\hline$\beta$ & $r_{I}$ & $a^{2} F\left(r_{I}\right)$ & $r / a$ & $a V(r)$ \\
\hline 0.75696 & 1.9233 & $0.3885(13)$ & 2 & $0.9004(17)$ \\
& 2.7793 & $0.2723(59)$ & 3 & $1.1726(33)$ \\
& 3.7435 & $0.241(10)$ & 4 & $1.410(11)$ \\
& 4.7364 & $0.2424(83)$ & 5 & $1.658(20)$ \\
& 5.7535 & $0.236(20)$ & 6 & $1.894(35)$ \\
\hline 0.8243 & 1.9233 & $0.29697(61)$ & 2 & $0.7357(11)$ \\
& 2.7793 & $0.1887(12)$ & 3 & $0.9244(11)$ \\
& 3.7435 & $0.1613(12)$ & 4 & $1.0857(17)$ \\
& 4.7364 & $0.1532(62)$ & 5 & $1.2389(70)$ \\
& 5.7535 & $0.1476(91)$ & 6 & $1.386(16)$ \\
& 6.7843 & $0.1494(61)$ & 7 & $1.536(20)$ \\
\hline 0.9636 & 1.9233 & $0.20810(27)$ & 2 & $0.55693(64)$ \\
& 2.7793 & $0.1136(16)$ & 3 & $0.6703(20)$ \\
& 3.7435 & $0.08680(85)$ & 4 & $0.7569(20)$ \\
& 4.7364 & $0.0783(13)$ & 5 & $0.8340(29)$ \\
& 5.7535 & $0.0745(37)$ & 6 & $0.9076(57)$ \\
& 6.7843 & $0.0700(57)$ & 7 & $0.977(10)$ \\
& 7.8120 & $0.0640(47)$ & 8 & $1.038(10)$ \\
\hline 1.04 & 1.9233 & $0.18182(26)$ & 2 & $0.49710(24)$ \\
& 2.7793 & $0.09392(54)$ & 3 & $0.59094(58)$ \\
& 3.7435 & $0.06847(84)$ & 4 & $0.65914(76)$ \\
& 4.7364 & $0.0575(18)$ & 5 & $0.7180(26)$ \\
& 5.7535 & $0.0544(12)$ & 6 & $0.7724(21)$ \\
& 6.7843 & $0.0527(22)$ & 7 & $0.8254(42)$ \\
& 7.8120 & $0.0498(23)$ & 8 & $0.8753(65)$ \\
& 8.8296 & $0.0481(42)$ & 9 & $0.9230(51)$ \\
& 9.8369 & $0.0485(44)$ & 10 & $0.9713(56)$ \\
& 10.8365 & $0.0464(34)$ & 11 & $1.0175(95)$ \\
& 11.8310 & $0.048(13)$ & 12 & $1.067(20)$ \\
\hline
\end{tabular}




\section{References}

[1] RBC, K. Orginos, Nucl. Phys. Proc. Suppl. 106 (2002) 721, hep-lat/0110074,

[2] Y. Aoki et al., (2002), hep-lat/0211023,

[3] K. Jansen and K.i. Nagai, (2003), hep-lat/0305009,

[4] CP-PACS, M. Okamoto et al., Phys. Rev. D60 (1999) 094510, heplat/9905005,

[5] R. Sommer, Nucl. Phys. B411 (1994) 839, hep-lat/9310022.

[6] Y. Iwasaki, UTHEP-118.

[7] QCD-TARO, P. de Forcrand et al., Nucl. Phys. Proc. Suppl. 53 (1997) 938, hep-lat/9608094,

[8] T. Takaishi, Phys. Rev. D54 (1996) 1050,

[9] P. Weisz, Nucl. Phys. B212 (1983) 1,

[10] P. Weisz and R. Wohlert, Nucl. Phys. B236 (1984) 397.

[11] P. Hasenfratz and F. Niedermayer, Nucl. Phys. B414 (1994) 785, heplat/9308004,

[12] F. Niedermayer, P. Rufenacht and U. Wenger, Nucl. Phys. B597 (2001) 413, hep-lat/0007007,

[13] A.M. Polyakov, Phys. Lett. B72 (1978) 477,

[14] L. Susskind, Phys. Rev. D20 (1979) 2610,

[15] G. Boyd et al., Nucl. Phys. B469 (1996) 419, hep-lat/9602007,

[16] B. Beinlich, F. Karsch, E. Laermann and A. Peikert, Eur. Phys. J. C6 (1999) 133, hep-lat/9707023,

[17] QCD-TARO, P. de Forcrand et al., Nucl. Phys. B577 (2000) 263, heplat/9911033,

[18] D.W. Bliss, K. Hornbostel and G.P. Lepage, (1996), hep-lat/9605041,

[19] M.J. Teper, (1998), hep-th/9812187, 
[20] ALPHA, M. Guagnelli, R. Sommer and H. Wittig, Nucl. Phys. B535 (1998) 389, hep-lat/9806005,

[21] S. Necco and R. Sommer, Nucl. Phys. B622 (2002) 328, hep-lat/0108008,

[22] APE, M. Albanese et al., Phys. Lett. 192B (1987) 163.

[23] G. Parisi, R. Petronzio and F. Rapuano, Phys. Lett. 128B (1983) 418.

[24] M. Creutz, Phys. Rev. D36 (1987) 515.

[25] F.R. Brown and T.J. Woch, Phys. Rev. Lett. 58 (1987) 2394.

[26] R.G. Edwards, U.M. Heller and T.R. Klassen, (1997), hep-lat/9711003.

[27] M. Luscher and P. Weisz, Nucl. Phys. B240 (1984) 349,

[28] N.A. Campbell, A. Huntley and C. Michael, Nucl. Phys. B306 (1988) 51.

[29] M. Lüscher and U. Wolff, Nucl. Phys. B339 (1990) 222.

[30] S. Necco and R. Sommer, Phys. Lett. B523 (2001) 135, hep-ph/0109093,

[31] B. Berg and A. Billoire, Nucl. Phys. B221 (1983) 109,

[32] U. Wenger, (2000), Ph.D.Thesis, unpub.,

[33] K. Ishikawa, G. Schierholz and M. Teper, Z. Phys. C19 (1983) 327,

[34] A. Vaccarino and D. Weingarten, Phys. Rev. D60 (1999) 114501, heplat/9910007,

[35] UKQCD, G.S. Bali et al., Phys. Lett. B309 (1993) 378, hep-lat/9304012,

[36] H. Wittig, (1999), hep-ph/9911400,

[37] C.J. Morningstar and M.J. Peardon, Phys. Rev. D60 (1999) 034509, heplat/9901004,

[38] M. Luscher and P. Weisz, JHEP 09 (2001) 010, hep-lat/0108014,

[39] H.B. Meyer, JHEP 01 (2003) 048, hep-lat/0209145,

[40] T. Blum et al., Nucl. Phys. B442 (1995) 301, hep-lat/9412038,

[41] JLQCD, S. Aoki et al., Nucl. Phys. Proc. Suppl. 106 (2002) 263, heplat $/ 0110088$, 